\title{
Malaria abrogates O'nyong-nyong virus pathologies by restricting virus infection in nonimmune cells
}

\author{
Anthony Torres-Ruesta ${ }^{1,2}$, Teck-Hui Teo ${ }^{1}$, Yi-Hao Chan ${ }^{1}$, Siti Naqiah Amrun ${ }^{1} \mathbb{0}$, Nicholas Kim-Wah Yeo $\mathbb{C}^{1}$, \\ Cheryl Yi-Pin Lee ${ }^{1}$, Samantha Yee-Teng Nguee ${ }^{1} \mathbb{\infty}$, Matthew Zirui Tay ${ }^{1}$, Francois Nosten ${ }^{3,4} \mathbb{C}$, Siew-Wai Fong ${ }^{1}$,

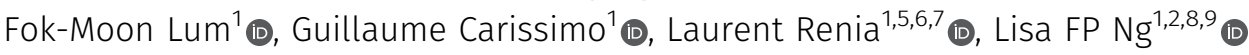

O'nyongnyong virus (ONNV) is a re-emerging alphavirus previously known to be transmitted by main malaria vectors, thus suggesting the possibility of coinfections with arboviruses in coendemic areas. However, the pathological outcomes of such infections remain unknown. Using murine coinfection models, we demonstrated that a preexisting blood-stage Plasmodium infection suppresses ONNV-induced pathologies. We further showed that suppression of viremia and virus dissemination are dependent on Plasmodium-induced IFN $\gamma$ and are associated with reduced infection of $\mathrm{CD} 5^{-}$cells at the site of virus inoculation. We further proved that treatment with IFNy or plasma samples from Plasmodium vivax-infected patients containing IFN $\gamma$ are able to restrict ONNV infection in human fibroblast, synoviocyte, skeletal muscle, and endothelial cell lines. Mechanistically, the role of IFNy in restricting ONNV infection was confirmed in in vitro infection assays through the generation of an IFN $\gamma$ receptor $1 \alpha$ chain (IFNYR1)-deficient cell line.

DOI 10.26508/Isa.202101272 | Received 23 October 2021 | Revised 4 January 2022 | Accepted 4 January 2022 | Published online 17 January 2022

\section{Introduction}

O'nyongnyong virus (ONNV) is an enveloped, positive-sense, singlestranded RNA virus that belongs to the Alphavirus genus of the Togaviridae family (1). It is closely related to other arthritogenic alphaviruses from the Semliki Forest antigen complex, such as chikungunya virus (CHIKV), Ross River virus (RRV), and Mayaro virus (MAYV) (2). ONNV pathology in humans is characterized by fever, maculopapular skin rash, myalgia, incapacitating polyarthralgia and to a lesser extent lymphadenopathy (1). The disease is generally self-limiting and resolves within some days, but symptoms such as polyarthralgia and myalgia may persist in a small proportion of the cases (3). ONNV was first isolated in 1959 in Gulu, Uganda (4), during an outbreak that lasted $3 \mathrm{yr}$ (1959-1962) and involved more than two million cases (5). After this outbreak, ONNV caused two other major epidemics: one in south-central Uganda in $1996(3,6)$ and another in Liberia and Ivory Coast in 2003 involving thousands of cases (7). More recently, epidemiological surveys have reported high seroprevalence of ONNV in Coastal Kenya (8) and Uganda (9), suggesting an underestimated burden of ONNV infections in sub-Saharan Africa.

The re-emergence and expansion of alphavirus infections in the tropics during the last decade introduce a new risk of coinfections with other highly prevalent endemic mosquito-borne diseases such as malaria. Limited serological studies in malaria-endemic countries in Africa have reported evidence of coinfections between Plasmodium parasites and alphaviruses. Specifically, anti-CHIKV antibodies were detected in a Nigerian cohort of Plasmodium falciparum-infected patients (10). In another study in Tanzania (11), a considerable proportion of febrile malaria patients were seropositive for $\mathrm{CHIKV}$, suggesting pre-exposure or active CHIKV infection. However, these reports should be interpreted cautiously because antibodies against CHIKV and ONNV are highly cross-reactive (9).

ONNV and Plasmodium parasites share common anopheline vectors such as Anopheles gambiae and Anopheles funestus (1), thus increasing the likelihood of co-transmission. However, reports on ONNV and Plasmodium coinfections in humans are lacking despite the increasing rates of ONNV transmission and the overwhelming presence of malaria in Sub-Saharan Africa. This, together with the highly inflammatory signature of both infections $(12,13,14$, $15,16)$, the detrimental role of $T$ cell-mediated immunity in the pathologies induced by both infections $(17,18,19,20,21)$, and the immunosuppressive nature of malaria (22, 23, 24, 25), strongly suggest that immune modulation could happen upon coinfection.

${ }^{1} A *$ STAR Infectious Diseases Labs (A*STAR ID Labs), Agency for Science, Technology and Research (A*STAR), Singapore, Singapore ${ }^{2}$ Department of Biochemistry, Yong Loo Lin School of Medicine, National University of Singapore, Singapore, Singapore ${ }^{3}$ Shoklo Malaria Research Unit, Mahidol-Oxford Tropical Medicine Research Unit (MORU), Faculty of Tropical Medicine, Mahidol University, Mae Sot, Thailand ${ }^{4}$ Nuffield Department of Medicine, Centre for Tropical Medicine and Global Health, University of Oxford, Oxford, UK ${ }^{5}$ Singapore Immunology Network, Agency for Science, Technology and Research (A*STAR), Singapore, Singapore ${ }^{6}$ Lee Kong Chian School of Medicine, Nanyang Technological University, Singapore, Singapore ${ }^{7}$ School of Biological Sciences, Nanyang Technological University, Singapore, Singapore ${ }^{8}$ National Institute of Health Research, Health Protection Research Unit in Emerging and Zoonotic Infections, University of Liverpool, Liverpool, UK ${ }^{9}$ Institute of Infection, Veterinary and Ecological Sciences, University of Liverpool, Liverpool, UK 
In this study, we describe the interactions between ONNV and rodent Plasmodium parasites in a mammalian host. Using mouse models of coinfections, we demonstrated that preexisting murine malaria restricts ONNV-associated pathologies and this protective effect is driven mainly by Plasmodium-induced IFNy by limiting ONNV infection in the CD45-cell compartment. In vitro experiments using human cell lines and plasma from Plasmodium vivaxinfected patients confirmed the antiviral role of IFNY in restricting ONNV infection. Our findings have potential implications in arbovirus and malaria control programs in endemic regions where Plasmodium parasites and arboviruses co-circulate.

\section{Results}

\section{Murine malaria suppresses ONNV-induced joint swelling and viremia}

An immunocompetent mouse model was previously established to recapitulate ONNV-induced joint pathologies (inflammation, edema, muscle necrosis, synovitis, and tenosynovitis) and acute viremia (26). Using this model, we first assessed whether a preexisting acute blood-stage Plasmodium infection could alter the development of ONNV pathologies. To do so, 3-wk-old C57BL/6] mice were inoculated with 1E6-infected red blood cells (iRBCs) from either Plasmodium berghei ANKA clone 231cl1 (PbA), which induces lethal neuropathology known as experimental cerebral malaria (ECM), or the nonlethal self-resolving strain Plasmodium yoelii 17XNL clone 1.1 (Py17x). When patent parasitemia was detected at $4 \mathrm{~d}$ post iRBC injection, 1E6 ONNV PFU were injected subcutaneously in the right paw. Viremia and joint swelling were measured for 12 and 14 days postinfection, respectively (dpi) (Fig 1A). The patent blood-stage Py17x infection protected coinfected animals from the development of ONNV-induced joint swelling (Figs 1B and S1A) and significantly reduced viremia levels (Fig 1C). Similarly, a preexisting blood-stage $\mathrm{PbA}$ infection was able to abolish virus-induced footpad swelling (Fig 1B). Of note, viremia in animals preinfected with $\mathrm{PbA}$ was undetectable during the entire follow-up suggesting a stronger protective effect by PbA than by Py17x against ONNV (Fig 1C).

To assess whether the suppression of viremia and ONNVinduced joint swelling by murine malaria was dependent on the timing of Plasmodium inoculation, two additional coinfection conditions were explored: concurrent coinfection and postviral coinfection. In concurrent coinfection, mice were coinfected with 1E6 ONNV PFU and 1E6 PbA or Py17x iRBC at the same time (Fig 1D). Concurrent coinfection did not affect the development of viremia in coinfected animals (Fig 1F) but had strain-specific effects on inflammation. Mice coinfected with Py17x did not display significant reduction in joint swelling, whereas mice coinfected with $\mathrm{PbA}$ displayed a significant reduction in joint swelling from $5 \mathrm{dpi}$ onwards with a major suppression at the peak of swelling at 6 dpi and an earlier resolution of the pathology at $10 \mathrm{dpi}$ compared with ONNV-infected controls (Figs $1 \mathrm{E}$ and S1B). Finally, we assessed postviral Plasmodium infection, where mice were infected with $1 \mathrm{E} 6$ PbA or Py17x iRBC $4 \mathrm{~d}$ after $1 \mathrm{E} 6$ ONNV PFU injection (Fig 1G). In this setting, no effect on ONNV-induced joint swelling (Figs 1H and S1C) or viremia (Fig 11) was observed, indicating that neither PbA nor Py17x infection could alter the course of a preexisting ONNV infection.

The effects of ONNV infection on the dynamics of malarial growth and survival were also assessed. The inoculation of ONNV $4 \mathrm{~d}$ post Py17x or PbA infection did not alter parasitemia levels (Fig S2A and B) or PbA-induced mortality (Fig S2B). Concurrent coinfection with nonlethal Py17x and ONNV resulted in increased parasitemia levels (Fig S2C). Py17x parasitemia resolution was delayed as coinfected animals took 26-28 d to clear blood-stage parasites compared with 20-22 d in controls. On the other hand, simultaneous inoculation with $\mathrm{PbA}$ and ONNV did not affect the development of parasitemia or ECM mortality in coinfected mice (Fig S2D). Finally, the infection with Plasmodium parasites $4 \mathrm{~d}$ post ONNV inoculation resulted in aggravated Py17x and PbA parasitemia (Fig S2E and F) but did not impact ECM mortality (Fig S2F).

\section{Early stages of ONNV replication in footpad tissues are impaired} in animals preinfected by Plasmodium parasites

As shown in Fig $1 \mathrm{C}$, a preexisting Plasmodium infection, either by $\mathrm{PbA}$ or Py17x, significantly reduced viremia in coinfected animals. To assess any possible differences in the kinetics of virus replication at the site of inoculation (right hind limb footpad), viral dissemination in vivo was assessed using a luciferase-tagged ONNV clone that mimics wild type ONNV infection in mice. Bioluminescence measurements from infected footpads correlate with viral burden in these tissues (26). ONNV-infected and coinfected animals were monitored during the first 24 hours postvirus inoculation (hpi) given that ONNV dissemination peaks at $12 \mathrm{hpi}$ (31). Significant differences in whole body and footpad radiance were detected as early as $1 \mathrm{hpi}$ in mice preinfected with PbA or Py17x and were maintained at 3, 6, 12, and $24 \mathrm{hpi}$ (Fig 2A-C and Video 1).

The absence of ONNV bioluminescence signals into adjacent tissues such as the tail (Fig 2C) in the coinfected mice prompted us to further assess ONNV dissemination into other major mouse tissues. Appendages (hind limb footpads and tail), internal organs (spleen, liver, and pLN) and muscle tissue (gastrocnemius and quadriceps) of ONNV-infected and coinfected animals were harvested at $1 \mathrm{dpi}$ and viral RNA was quantified. Lower viral loads were observed in most of the tissues assessed, with major differences occurring in organs distant from the site of inoculation such as liver and spleen where viral burden in coinfected mice were on average $\approx 10,000$-fold and $\approx 1,000$-fold lower, respectively (Fig 2D).

\section{Preexisting Plasmodium infection renders $\mathrm{CD} 45^{+}$and $\mathrm{CD}_{4} 5^{-}$ footpad cells less susceptible to ONNV infection}

Our data strongly suggested that a preexisting blood-stage Plasmodium infection renders footpad cells less susceptible to ONNV. To identify the cellular subsets involved in the suppression of ONNV infection, we defined the differences in ONNV infection of nonimmune $\left(C D 45^{-}\right)$and immune $\left(C D 45^{+}\right)$cells in footpads of coinfected mice at $12 \mathrm{hpi}$ (peak of footpad viral load in Fig 2B). For this purpose, a ZsGreen-tagged ONNV infectious clone detectable by flow cytometry under the FITC channel was used. 

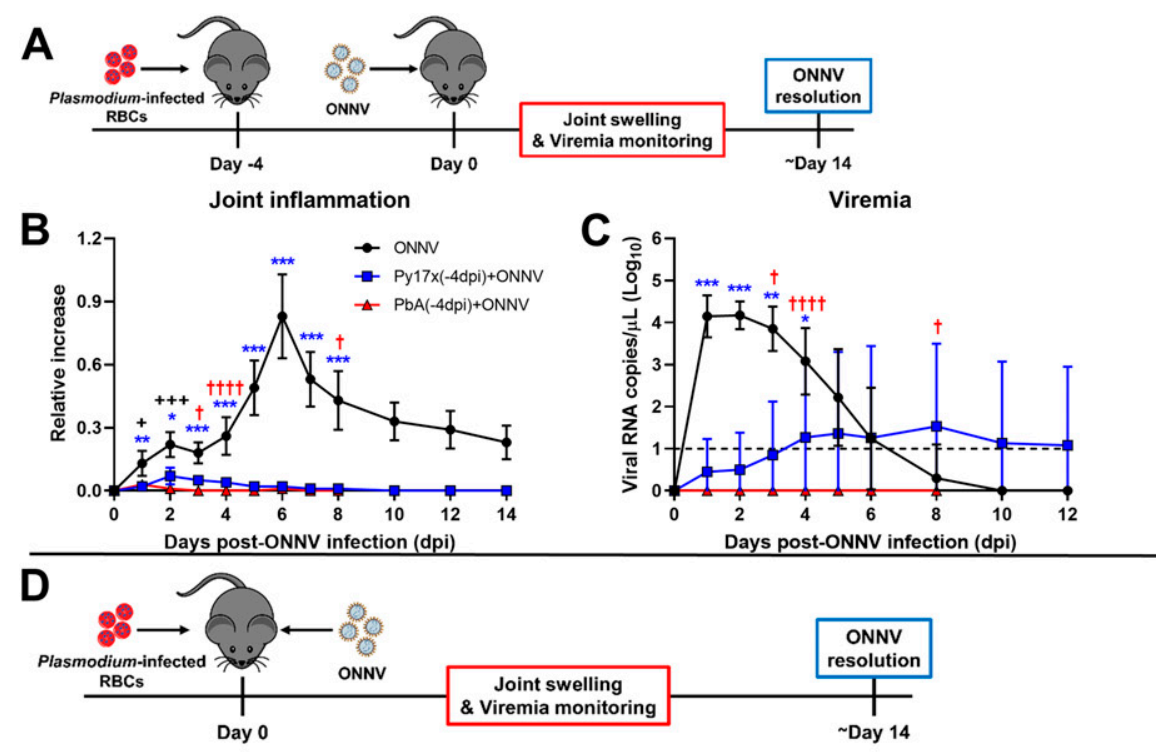

E
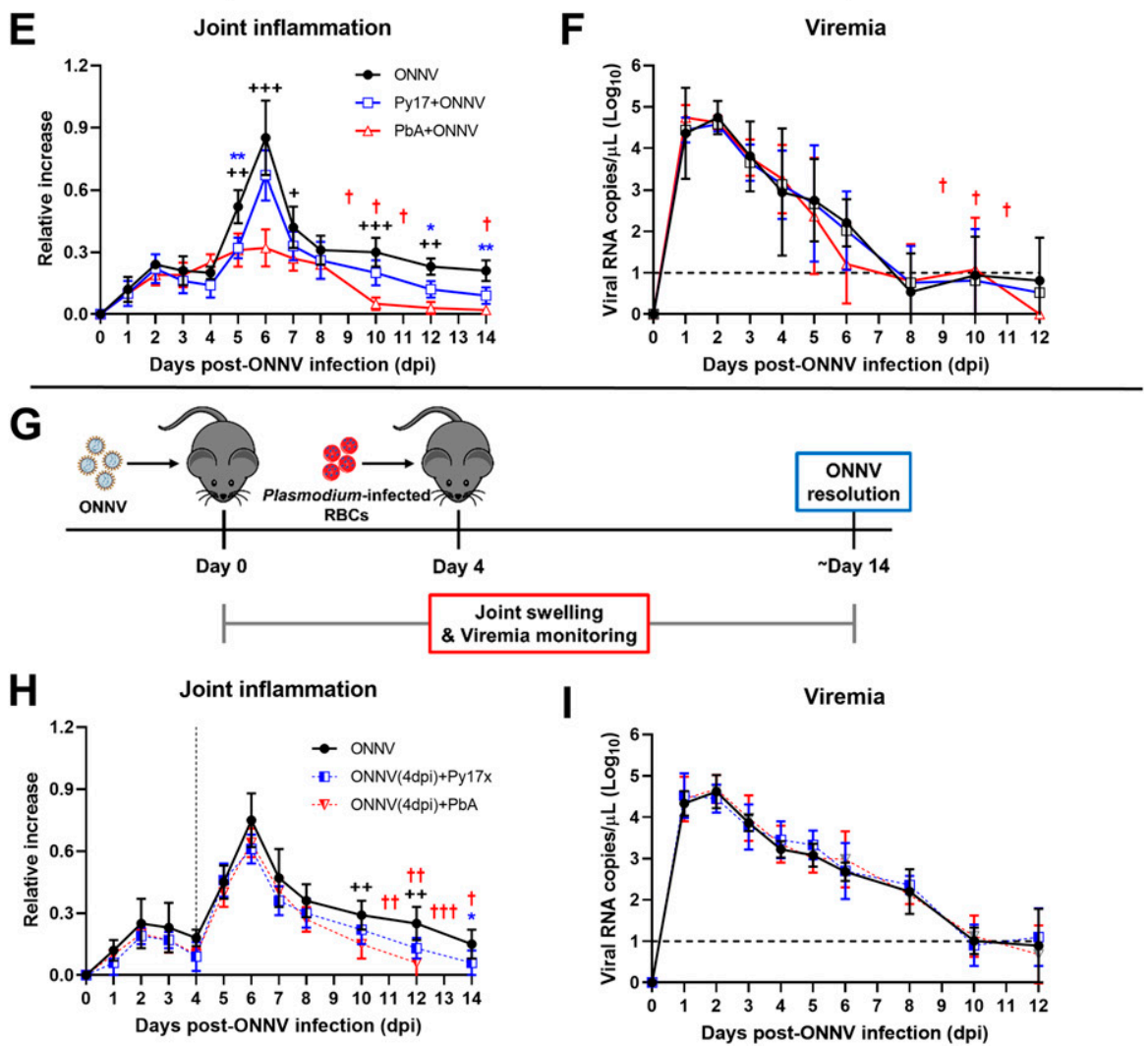

Figure 1. Preexisting murine malaria protects mice from ONNV-induced pathologies.

(A) Previral Plasmodium infection: mice were infected with $\mathrm{PbA}$ or Py17x $4 \mathrm{~d}$ prior ONNV inoculation according to the schematic in (A). (B, C) Joint swelling and (C) viremia measurements in ONNV, Py17x(-4dpi)+ONNV, and $\mathrm{PbA}(-4 \mathrm{dpi})+\mathrm{ONNV}$ groups. (D) Concurrent coinfection: animals were simultaneously infected with ONNV and PbA or Py $17 x$ on the same day according to the schematic in (D). (E, F) Joint swelling and (F) viremia measurements in ONNV, Py17X+ONNV, and $\mathrm{PbA}+$ ONNV groups. (G) Postviral Plasmodium infection: mice were infected with ONNV $4 \mathrm{~d}$ prior PbA or Py17x inoculation according to the schematic in $(\mathrm{G})$.

$(\mathbf{H}, \mathbf{I})$ Joint swelling and (I) viremia measurements in ONNV, ONNV(-4dpi)+Py17x, and ONNV(-4dpi)+PbA groups. Data are presented as mean \pm SD of at least five animals per experimental group and are representative of two independent experiments. Differences between ONNV controls and coinfected mice with $\mathrm{PbA}(++P<0.01$ $\left.{ }^{+++} P<0.01\right)$ or Py17x $\left({ }^{*} P<0.05,{ }^{* *} P<0.01,{ }^{* * *} P<0.001\right)$ were calculated using two-tailed Kruskal-Wallis and post hoc Dunn's tests. When PbA-infected animals succumbed to ECM, differences between ONNV singly infected controls and coinfected mice with Py17x were computed using two-tailed Mann-Whitney $U$ test instead. " + " represents one mouse that succumbed of $\mathrm{PbA}$-induced ECM on the respective day. Horizontal dashed lines in (C), (F) and (I) represent the qRT-PCR detection limit. Vertical dashed line in $(\mathrm{H})$ represents the day on which Plasmodium parasites were inoculated.
To understand the individual contribution of $\mathrm{CD} 45^{+}$and $\mathrm{CD} 45^{-}$ compartments to the total ONNV-infected cells at $12 \mathrm{hpi}$, the infectivity profile of footpads was analyzed in ONNV-infected mice. It was observed that $\sim 80 \%$ of the ONNV-infected footpad cells at 12 hpi were part of the $\mathrm{CD}_{4} 5^{-}$nonimmune compartment (Fig S3A). When the analysis was extended to median fluorescence intensity values, CD45 cells appeared to harbour on average a higher number of ONNV-ZsGreen copies than CD45 cells (Fig S3B). These observations highlight a major role of $\mathrm{CD}^{-} 5^{-}$cells as an early target for ONNV replication.

The impact of coinfections on the infectivity rates of $\mathrm{CD}_{4} 5^{+}$and $\mathrm{CD} 45^{-}$cells was then assessed. Animals preinfected with either $\mathrm{PbA}$ or Py17x displayed a marked reduction in the percentage and total counts of $\mathrm{CD} 45^{+} \mathrm{ZsGreen}{ }^{+}$and $\mathrm{CD} 5^{-} \mathrm{ZsGreen}^{+}$cells compared with ONNV controls (Fig S3C and D). Given the importance of nonimmune cells as alphavirus targets during the early stages of infection 

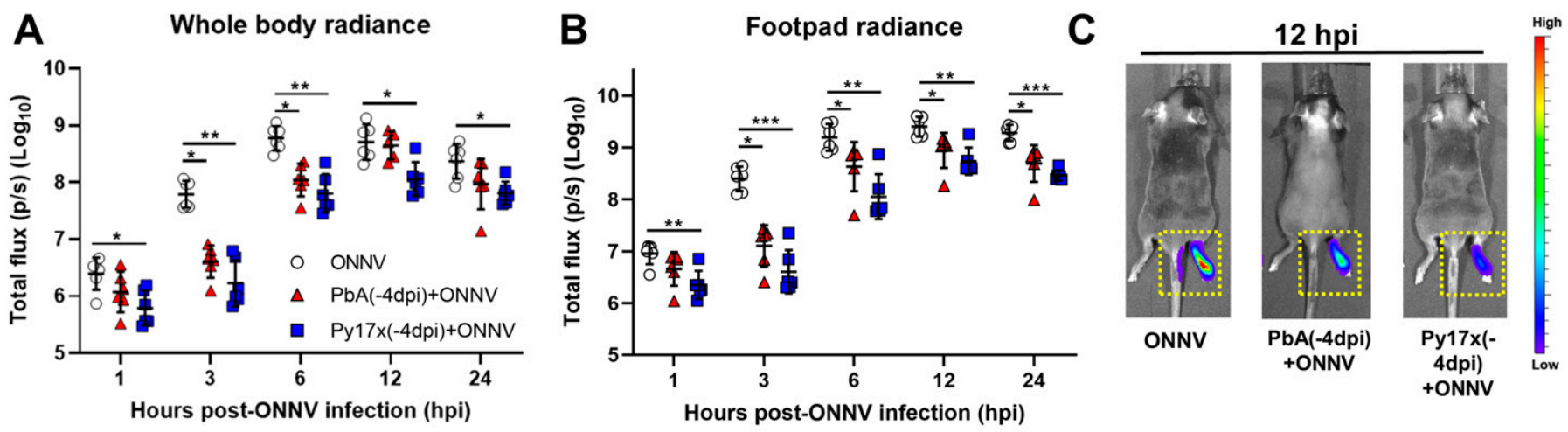

D

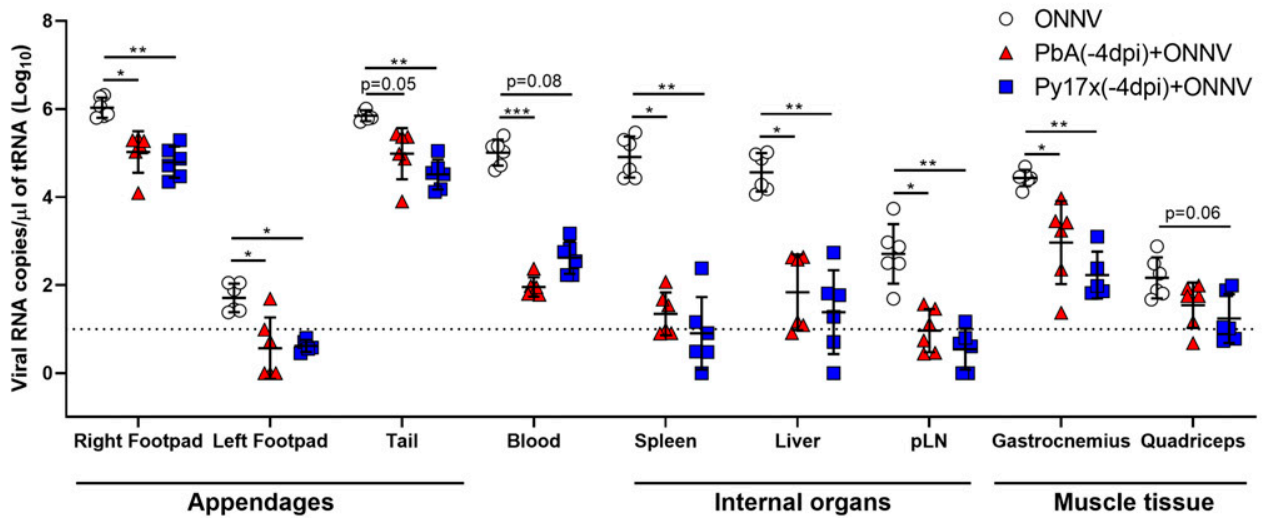

Figure 2. Early stages of ONNV replication and dissemination are supressed by preexisting Plasmodium infection.

Mice were infected with Py $17 x$ or PbA and $4 \mathrm{~d}$ postinfection were inoculated with a firefly luciferase-tagged ONNV clone in the right hind limb footpad. Before data acquisition, mice were injected with $100 \mu \mathrm{l}$ of D-Luciferin $(5 \mathrm{mg} / \mathrm{ml}$ ) subcutaneously. (A, B) Whole body radiance and (B) footpad radiance of ONNV, Py17x(-4dpi)+ONNV, and $\mathrm{PbA}(-4 \mathrm{dpi})+\mathrm{ONNV}$ groups at 1, 3, 6, 12, and $24 \mathrm{hpi}$. (C) Representative pseudo-coloured images of bioluminescence readings depicting reduction of tissue viral load and restriction of viral dissemination in coinfected animals at $12 \mathrm{hpi}$ (yellow-doted boxes). (D) Tissue viral load in mouse appendages, internal organs and muscle detected by qRT-PCR at $24 \mathrm{hpi}$. Data are presented as mean \pm SD of at least five animals per experimental group. Differences between ONNV controls and coinfected mice with PbA or Py17x were calculated using two-tailed Kruskal-Wallis and post hoc Dunn's tests $\left({ }^{*} P<0.05{ }^{* *} P<0.01,{ }^{* * *} P<0.001\right)$.

$(27,28,29)$, the differences in infectivity within the $\mathrm{CD}_{4} 5^{-}$compartment were further characterized using Uniform Manifold Approximation and Projection (UMAP) on the flow cytometry data. Manually gated $\mathrm{CD} 45^{-}$populations based on the expression of signature surface markers $(30,31,32,33,34)$ were overlapped in the UMAP plot and allowed the identification of four major cell lineages: endothelial cells $\left(\mathrm{CD} 45^{-} \mathrm{CD} 29^{+} \mathrm{CD} 31^{+}\right)$, myoblasts $\left(\mathrm{CD} 45^{-} \mathrm{CD} 31^{-}\right.$Sca-1 $\left.{ }^{+}\right)$, fibroblasts ( $\left.\mathrm{CD}^{-} 5^{-} \mathrm{CD}^{+} \mathrm{CD}^{2} 9^{+} \mathrm{CD} 31^{-} \mathrm{Sca}-1^{-}\right)$, and mesenchymal stem cells (CD45 $\mathrm{CD}^{-} \mathrm{CD} 29^{-} \mathrm{CD} 31^{-} \mathrm{SCa}-1^{-}$) (Fig 3A). UMAP plots displaying the distribution of ZsGreen+ events in ONNV-infected and coinfected mice are also shown in Fig $3 \mathrm{~A}$.

As observed from the UMAP plots, ONNV infection was globally suppressed in endothelial cells, myoblasts, and fibroblasts from coinfected animals at $12 \mathrm{hpi}$ (Fig 3A). A similar trend was observed when $\mathrm{ZsGreen}^{+}$cells were quantified revealing a reduced number of infected endothelial cells, myoblasts, and fibroblasts (Fig 3B). To explore whether the reduced infectivity in $\mathrm{CD}_{4} 5^{-}$cells was a transient or maintained effect beyond $12 \mathrm{hpi}$, footpads from coinfected mice with Py17x were harvested at 48 and 72 hpi and analyzed by flow cytometry. Coinfected mice with PbA were not included in these sets of experiments because of the high mortality rate induced by ECM. Interestingly, suppression of ONNV infection in coinfected animals was observed across all the different time points assessed, suggesting a sustained suppression of viral load and not a delay in viral load appearance (Fig 3C).

\section{Murine malaria up-regulates proinflammatory immune mediators in footpad tissues}

Acute Plasmodium infections are known to induce strong proinflammatory responses characterized by the production and release of cytokines/chemokines and other mediators into the bloodstream to control parasite burden $(15,16)$. Thus, we hypothesized that some of these soluble factors could be responsible for the decreased susceptibility to ONNV infection observed in footpad tissues.

36 different immune mediators were assessed in sera (Fig 4A) and footpad tissue lysates (Fig 4B) of mice singly infected with lethal PbA or self-resolving Py17X at $4 \mathrm{~d}$ postparasite infection (time of ONNV inoculation in the coinfection model). Interestingly, results 


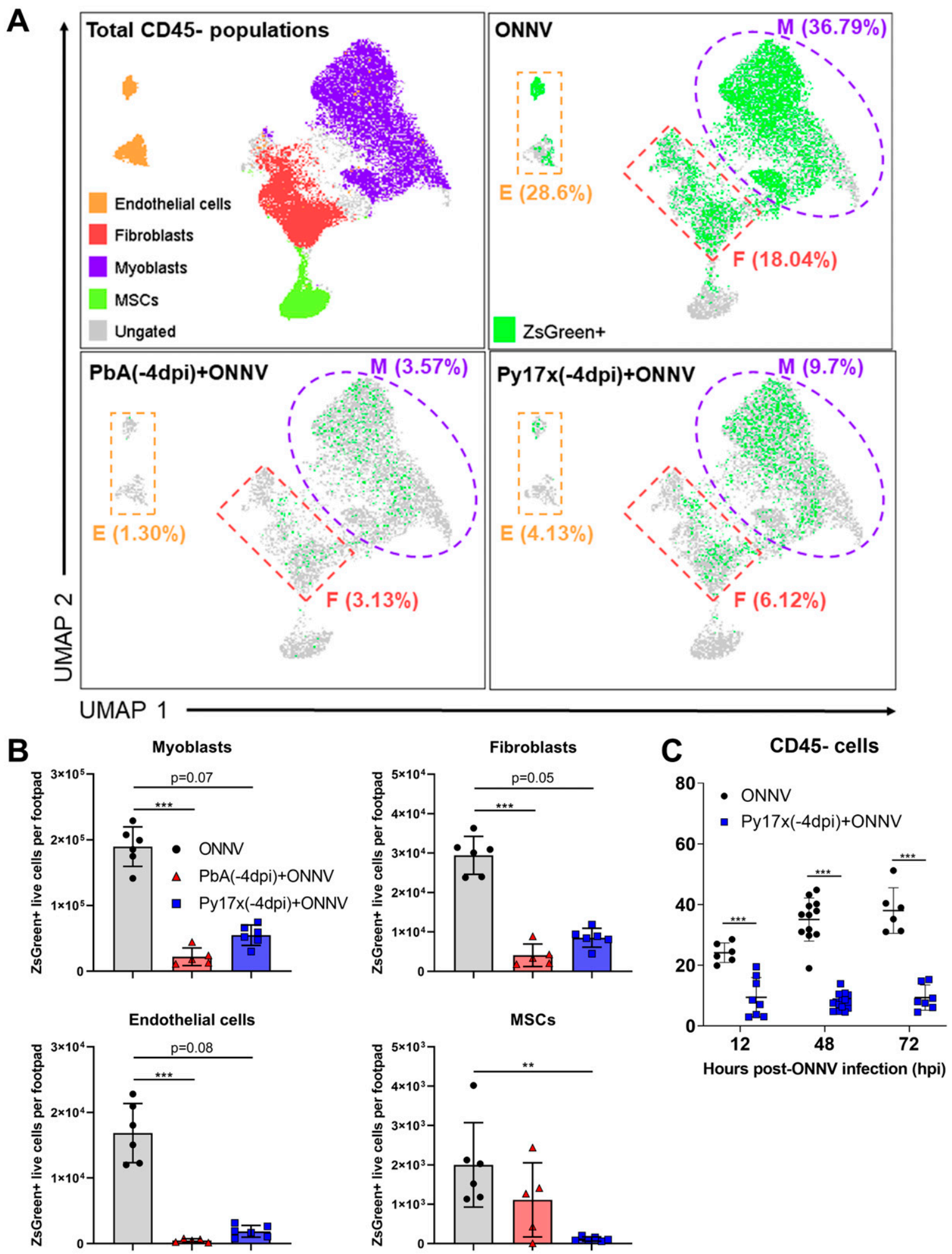

Figure 3. Prior Plasmodium infection restricts ONNV replication in $\mathrm{CD}_{4} 5^{-}$footpad cells.

(A) UMAP analysis of 105,000 live CD45- footpad cells from naive, ONNV, PbA(-4dpi)+ONNV, and Py17x(-4dpi)+ONNV groups at 12 hpi. The UMAP plot was generated by concatenation of samples containing 5,000 randomly selected live CD45 cells from each sample. ONNV, PbA(-4dpi)+ONNV, and Py17X(-4dpi)+ONNV UMAP plots show the global distribution of ZsGreen+ events (ONNV-infected cells). Colored dashed boxes highlight myoblasts (M), fibroblasts (F), and endothelial cells (E) and median ONNV infection rates per population. (B) Total counts of ONNV-ZsGreen+ cells in endothelial cells, myoblasts, fibroblasts and mesenchymal stem cells (MSCS) in ONNV, $\mathrm{PbA}(-4 \mathrm{dpi})+\mathrm{ONNV}$, and Py17x(-4dpi)+ONNV at 12 hpi. (C) Percentages of infected CD45 cells in ONNV and Py17x(-4DPI)+ONNV groups at 12, 48, and 72 hpi. Data are 
showed that PbA- and Py17x-infected mice generated distinct proinflammatory cytokine profiles. Using principal component analysis on these cytokine profiles, a distinct separation was revealed between samples from mock (green), PbA-infected (red), and Py17xinfected mice (green) (Fig 4C).

Levels of pro-inflammatory IFNy were increased by 292- and 28-folds in sera from PbA and Py17x-infected mice, respectively (Fig 4D), in line with previous reports $(35,36,37)$. Up-regulation of chemokines such as CXCL10 (IFNy-induced protein 10: IP-10), CXCL1, CCL2, CCL3, CCL5, and CCL7 was also observed in the sera (Fig 4D). Locally at the footpad, changes in immune mediator profiles were characterized by increased chemokine production, particularly IFNy-induced CXCL10, which was found to be elevated by 20- and 11-folds in tissue lysates of PbA and Py17x-infected animals, respectively (Fig 4D). Other up-regulated chemokines shared between $\mathrm{PbA}$ and Py17x-infected mice were CCL2, CCL3, CCL4, CCL5, CCL7, and CCL11. In addition, IL-18 (IFNy inducing factor: IGIF) and IFNy were found to be slightly elevated in footpad tissues upon PbA and Py17x infection (Fig 4D).

Differentially regulated cytokines/chemokines in the footpads were subjected to STRING analysis (performed with a high confidence threshold of 0.9), revealing key interactions between eight of these immune mediators (Fig S4). The predicted interactions are linked to cellular responses to IFNy, host-negative regulation of viral transcription and leukocyte recruitment and activation. Collectively, these data suggest that Plasmodium infection (either by PbA or Py17x) triggers a systemic immune response characterized by the up-regulation of pro-inflammatory immune mediators not only in the blood but also locally in footpad tissues. Importantly, the increased levels of immune mediators linked to IFNY signaling, a known antiviral cytokine (38), in footpad lysates of Plasmodiuminfected mice suggested a possible involvement of this cytokine in the restriction of ONNV infection.

\section{Plasmodium-induced IFN $\gamma$ mediates the suppression of ONNV replication and dissemination in coinfected animals}

To assess the potential role of IFNy in suppressing ONNV pathologies, IFNy-deficient animals were infected with nonlethal Py17x. $4 \mathrm{~d}$ postinfection, luciferase-tagged ONNV was inoculated subcutaneously in the right footpad. Lack of IFNy in coinfected animals abolished the antiviral effects exerted by Py17x infection in mice. Bioluminescence readings at 3, 6, 12, and 24 hpi were comparable with those from IFNY-deficient animals singly infected with ONNV (Fig 5A and Video 2). Similarly, ONNV viremia in coinfected IFNYdeficient mice was also restored (Fig 5B) despite a suppression of joint swelling at $6 \mathrm{dpi}$ (Fig S5). This suggests that coinfection might modulate other immune responses leading to joint swelling suppression in the absence of IFNy.

Because nonimmune cells express IFNy receptors (38) and can respond to IFNy signaling, the infection profile of these subsets was assessed in coinfected mice deficient in IFNy. Likewise, ONNV infectivity profiles of $\mathrm{CD}_{4} 5^{-}$cells were restored in myoblasts, endothelial cells, fibroblasts, and mesenchymal stem cells (Fig 5C and D). To further validate these findings, in vivo IFNy neutralization was performed using anti-mouse IFNy antibodies. Coinfected animals treated with antimouse IFNy displayed comparable ONNV tissue viral loads at 3, 6, 12, and 24 hpi (Fig 5E and Video 3) than isotype-control treated mice, suggesting a major role of IFNy in the antiviral effects exerted by preexisting Plasmodium infections.

Blood-stage Plasmodium infections in humans and mice are known to induce the production of type I IFN responses $(39,40,41)$. Type I IFN is a major regulator of susceptibility to alphavirus infection $(13,42,43)$ and evidence has suggested crosstalk mechanisms with IFNy signaling $(44,45,46)$. To evaluate any possible contribution of type I IFN responses in the reduced susceptibility to ONNV upon coinfection, the effect of preexisting murine malaria on ONNV replication was assessed in IFNaR ${ }^{-/-}$mice (deficient of IFN$\alpha / \beta$ receptor). Viremia measurements at 12,24 , and $48 \mathrm{hpi}$ in coinfected IFNaR ${ }^{-/-}$mice (Fig S6) revealed that murine malaria was still able to restrict ONNV infection, ruling out the involvement of type I IFN responses in the antiviral effects exerted by Plasmodiuminduced IFNY.

\section{IFN $\gamma$ inhibits ONNV infection in human fibroblast, synoviocyte, skeletal muscle, and endothelial cell lines}

The antiviral role of IFNy in nonimmune cell lineages upon ONNV infection remains poorly defined. To extrapolate our findings in the human context, the antiviral effect of IFNy was assessed in human cell lines representing skin fibroblasts (BJ), synoviocytes (SW982), endothelial cells (HPMECS), and skeletal muscle cells (RD) in an in vitro infection system. Interestingly, each cell type displayed different susceptibility to ONNV infection. Particularly, skin fibroblast and synoviocyte cell lines are highly susceptible to ONNV, whereas endothelial cells and skeletal muscle cells are poorly infected. Nevertheless, regardless of cell type, IFNy pretreatment successfully reduced ONNV infection in BJ, SW982, HPMEC, and RD cells at 24, 48, and 72 hpi in a dose-dependent manner (Fig 6A).

Finally, we characterized the ability of plasma samples from acute $P$. vivax-infected patients to render human cell lines less susceptible to ONNV infection. Plasma IFNy levels were quantified, and samples were categorized and pooled into low $(n=13$, median IFNy concentration $=69.53 \mathrm{pg} / \mathrm{ml})$ and high IFNy producers $(n=14$, median IFNy concentration $=293.675 \mathrm{pg} / \mathrm{ml}$ ). 10 plasma samples from healthy individuals were pooled and included in the experiments as controls (IFNy concentration under the quantification limit) (Fig 6B). Incubation of skin fibroblasts (BJ) with plasma from either low or high IFNy producers reduced ONNV infection compared with those treated with healthy control plasma (Fig 6C). To prove that IFNy present in the plasma samples from acute $P$. vivax-infected patients was responsible for the antiviral effects exerted in vitro, we generated a HEK293T cell line with impaired IFNY signaling (Fig S7A and B) by knocking down the expression of the IFNy receptor $1 \alpha$ chain (IFNYR1). Upon treatment with plasma from malaria patients, HEK293T cells with intact IFNYR1 expression

presented as mean \pm SD of at least five animals per experimental group. Differences between ONNV controls and coinfected mice with PbA or Py17x were calculated using two-tailed Kruskal-Wallis and post hoc Dunn's tests $\left({ }^{* *} P<0.01,{ }^{* * *} P<0.001\right)$. 

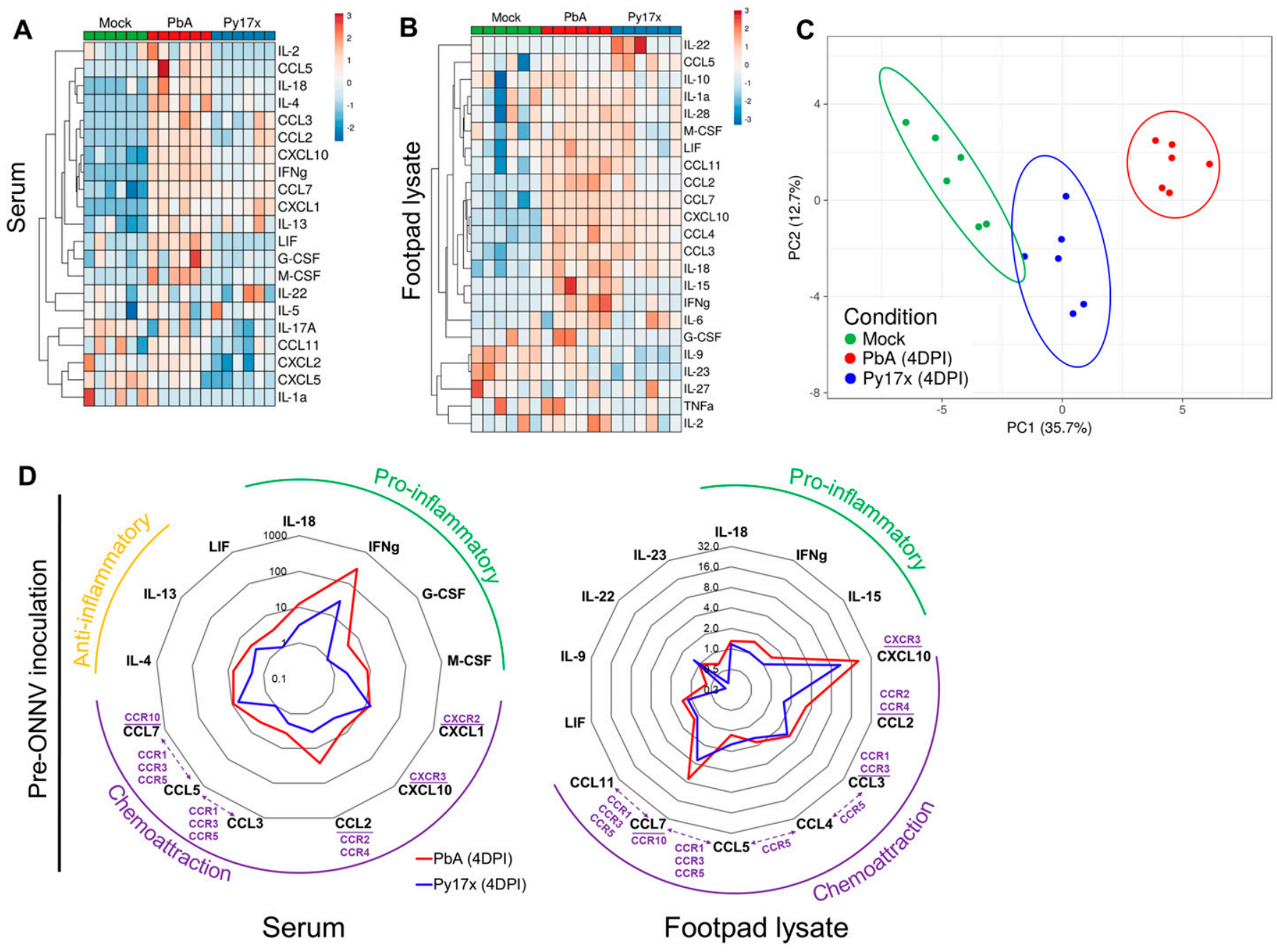

Figure 4. Footpads of Plasmodium-infected mice display a pro-inflammatory milieu.

(A, B) Heat map plots showing the detected cytokines/chemokines in (A) serum and (B) footpad lysates of mock-infected (green), PbA-infected (red), and Py17x-infected (blue) mice at $4 \mathrm{dpi}$. Analyte concentrations $(\mathrm{pg} / \mathrm{ml}+1)$ were logarithmically transformed $\left(\log _{10}\right)$ and Z-scores were calculated for representation purposes. Principal component analysis (PCA) and heat map plots were constructed using ClustVis. (C) PCA using differentially expressed analytes in footpad lysates and sera of mock, PbAinfected ( 4 dpi), and Py17x-infected ( 4 dpi) groups. PCA plot shows that PC1 (responsible for $35.7 \%$ of the variation) and PC2 (responsible for $12.7 \%$ of the variation) segregate the populations in three clusters: mock (green), PbA-infected (red), and Py17x-infected (blue). Colored ellipses were calculated with $95 \%$ confidence levels. (D) Radar plots showing median fold changes of differentially expressed cytokines/chemokines in serum and footpad lysates of PbA-infected (4 dpi) and Py17x-infected ( $4 \mathrm{dpi}$ ) groups relative to mock animals. Each cytokine/chemokine is grouped according to its immunological function (green: pro-inflammatory, yellow: antiinflammatory) or homing receptors (purple) as indicated. Shared chemokine receptors are shown in dashed lines. Data correspond six animals per experimental group. Differences between naïe, PbA, or Py17x-infected mice calculated using two-tailed Kruskal-Wallis and post hoc Dunn's tests.

displayed lower ONNV infection compared with untreated controls. This antiviral effect was lost in cells with impaired IFNYR1 expression ( $\triangle$ IFNyR1) (Fig 6D), in agreement with an IFNy-specific effect.

\section{Discussion}

O'nyongnyong virus and Plasmodium parasites share common anopheline vectors and co-circulate in sub-Saharan Africa with risk of human coinfection. This is the first study investigating the pathological outcomes of coinfection by Plasmodium parasites and alphavirus ONNV in a mammalian host. Here, we showed that a preexisting murine Plasmodium infection is able to suppress the development of ONNV pathologies by restricting viral infection at the site of inoculation and dissemination to distant organs. We demonstrated that Plasmodium-induced IFNy is the main cytokine driving the antiviral effects observed.

IFNy is a pleiotropic cytokine known for its ability to regulate immune responses by promoting macrophage activation, enhancing antigen presentation, modulating helper $\mathrm{T}$ cell development and mediating viral and bacterial immunity, among others (47). In ONNV-infected animals, it was observed that nearly $80 \%$ of the total virus-infected cells at 12 hpi (Fig S3A) belonged to the $\mathrm{CD}^{-} 5^{-}$compartment corroborating previous observations from other closely related alphaviruses, whereby nonimmune cells support the early stages of viral replication (13, 27, 28, 29, 48). In contrast, animals harboring a Plasmodium infection displayed 
A

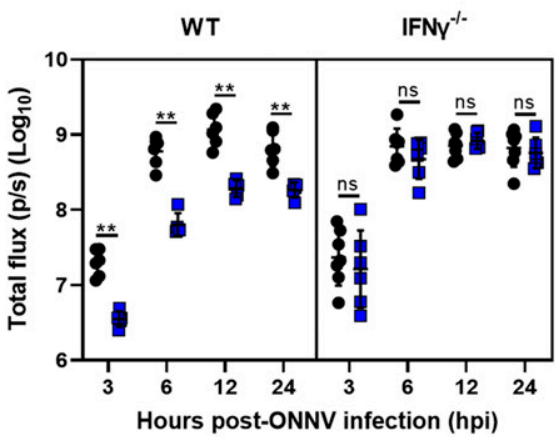

Footpad radiance

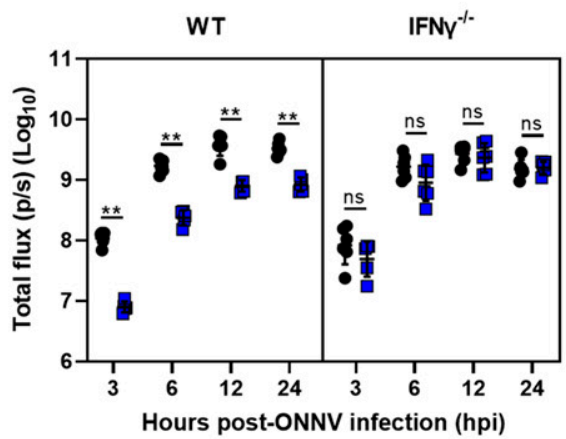

B

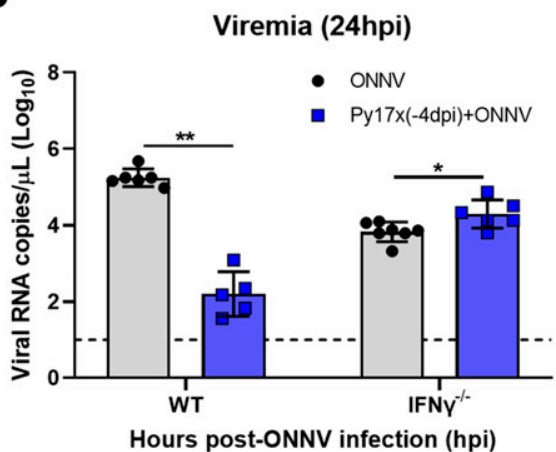

C
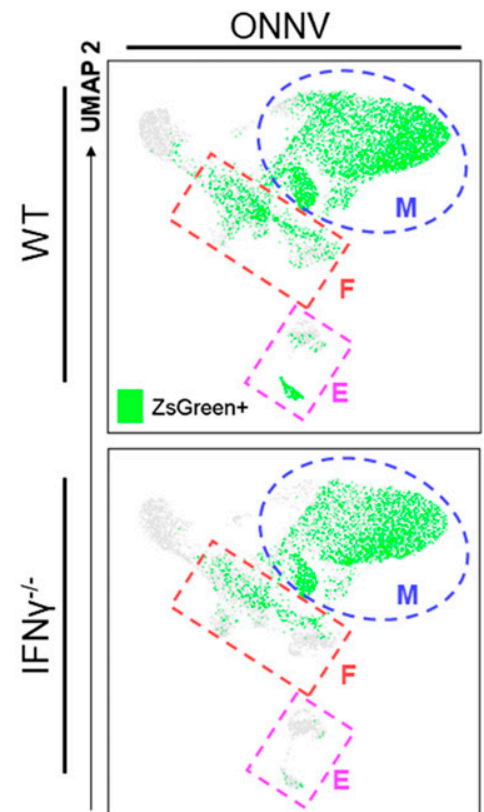

PbA(-4dpi)+ONNV Py17x(-4dpi)+ONNV
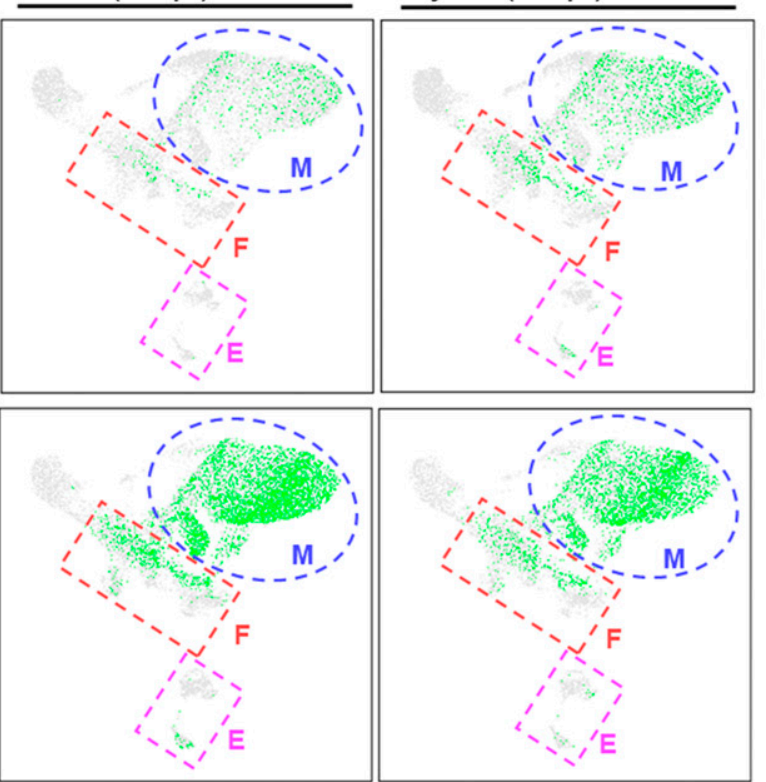

Total CD45- populations

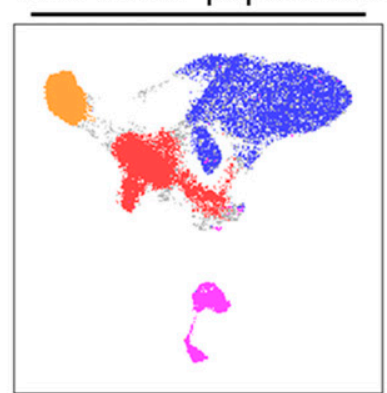

Endothelial cells

MSCs

Fibroblasts

Myoblasts

Undetermined

$\rightarrow$ UMAP 1
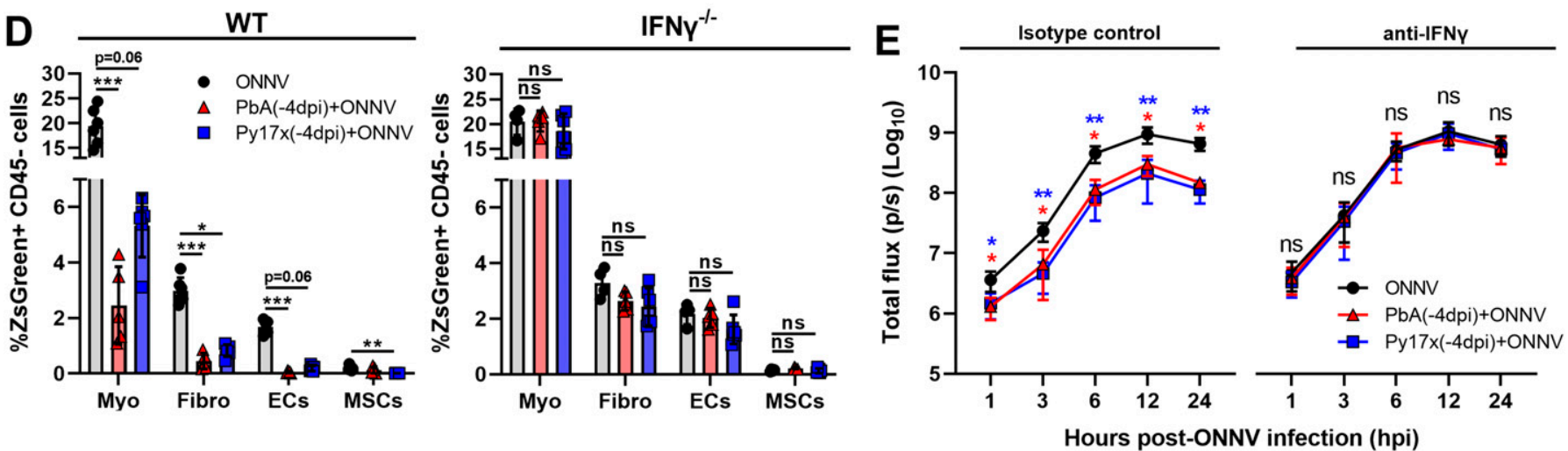

Figure 5. Plasmodium-induced IFNy mediates the suppression of ONNV replication and dissemination in coinfected animals.

(A, B) In vivo luminescence readings of (A) whole body and footpad radiance at 3, 6, 12, and $24 \mathrm{hpi}$ and (B) viremia at $24 \mathrm{hpi}$ of ONNV and Py17x(-4dpi)+ONNV wild-type (WT), or IFNY-deficient (IFNY ${ }^{-1-}$ ) animals. (C) UMAP analysis of 160,000 live CD45 footpad cells from WT or IFN ${ }^{-1-}$ ONNV, PbA(-4dpi)+ONNV, and Py17X(-4dpi)+ONNV groups at $12 \mathrm{hpi}$. The UMAP plot was generated by concatenation of samples containing 5,000 randomly selected live CD $45^{-}$cells from each sample. ONNV, PbA(-4dpi)+ONNV, and Py17x(-4dpi)+ONNV UMAP plots show the global distribution of ZsGreen+ events (ONNV-infected cells). Colored dashed boxes highlight ONNV infection in myoblasts, M; fibroblasts, F and endothelial cells, E. (D) Frequency of CD45-ZsGreen+ footpad cells of WT or IFNy ${ }^{-1-}$ ONNV, PbA(-4dpi)+ONNV, and Py17x(-4dpi)+ONNV groups at 12 hpi. Myo, myoblasts; Fibro, fibroblasts; ECS, endothelial cells and MSCS, mesenchymal stem cells. (E) Footpad radiance at 1, 3, 6, 12, and 24 hpi of ONNV, PbA(-4dpi)+ONNV, and Py17x(-4dpi)+ONNV groups in animals treated with mouse anti-IFNy or isotype control. Data are presented as mean \pm SD of at least five animals per experimental group. (A, B) Two-tailed Mann-Whitney $U$ test was used to compute differences between ONNV and Py17x $(-4 \mathrm{dpi})+$ ONNV groups in $(\mathrm{A})$ and $(B)\left({ }^{*} P<0.05{ }^{* *} P<0.01\right)$. Differences 
reduced numbers of ONNV-infected myoblasts, fibroblasts and endothelial cells and this protective effect was reverted in IFNy-deficient mice or upon in vivo IFNy neutralization. We therefore hypothesize that production of IFNy in response to acute blood-stage Plasmodium infection could stimulate cells from the $\mathrm{CD} 45^{-}$compartment activating antiviral processes (Fig S4) which in turn restrict a subsequent ONNV infection. In line with this, the antiviral effects of IFNy have been observed in other alphaviruses such as Sindbis virus (SINV). In vitro studies suggested that IFNY affects SINV replication in mature neurons by interfering with the synthesis of genomic and sub-genomic viral RNA (49) and that this effect is dependent on JAK/ STAT signaling (50). The contribution of Plasmodium-induced type I IFN $(40,41)$ to the reduced susceptibility to ONNV infection was also assessed in $\mathrm{IFNaR}^{-1-}$ mice. Considerable viremia differences were observed between ONNV-infected wild type and $\mathrm{IFNaR}^{-1-}$ controls $\left(\sim 4-5 \log _{10}\right.$ at $\left.48 \mathrm{hpi}\right)$ highlighting the importance of IFN- $\alpha / \beta$ signaling in the control of ONNV infection as observed in other alphavirus animal models $(42,43,51,52)$. Nonetheless, type I IFNinduced upon Plasmodium infection seems to be negligible for the establishment of protective effects by murine malaria as coinfected $\mathrm{IFNaR}^{-1-}$ mice still displayed reduced ONNV infection to a comparable level than coinfected wild-type mice (Fig S6).

It is important to note that in our experiments, mice only experienced suppression of ONNV viremia and virus dissemination after $4 \mathrm{~d}$ post-Plasmodium inoculation (Figs 1B and 2A-D) and not upon concurrent or sequential (postviral) coinfection. These observations strongly suggested that the timing of parasite inoculation and induction of IFNY are critical for the protective effects to happen. Interestingly, although the main suppression of ONNV infection occurred in joint footpad cells, we observed lower concentrations of IFNy in joint footpad tissues compared with serum samples at $4 \mathrm{~d}$ post-Plasmodium inoculation. Thus, it is likely that IFNy levels in joint footpads could have increased at an earlier time point. In support of this, we observed high concentrations of IFNyinduced immune mediators in joint footpad tissues, particularly CXCL10 (53) and CCL7, known to be produced by fibroblasts and mononuclear cells upon IFNy stimulation (54). The development of T-cell responses, major IFNy-producing subsets during malaria (ref), could also influence the outcome of a Plasmodium-ONNV coinfection in murine models. Early in a blood-stage infection, a large number of IFNy-secreting Th1 cells are produced, whereas Th2-like responses govern during the chronic phase of infection (17). Because ONNV inoculation occurs in the early stages of murine malaria ( $4 \mathrm{dpi}$ ), it is likely that the antiviral effects of IFNy are associated to the establishment of Th1 immunity against the parasite. It can be speculated that the degree of virus suppression might differ if ONNV is inoculated during the chronic stage of the murine malaria, particularly when Th1 responses are weaning.

We explored the relevance of our findings in the context of ONNV human infection by treating four human cell lines from different nonimmune lineages (fibroblast, synoviocyte, endothelial, and skeletal muscle cells) with IFNy before ONNV infection. Mechanistically, our results demonstrated that IFNY is able of restricting ONNV infection in human cell lines in a dose dependent manner. Similarly, we also observed that stimulation of skin fibroblasts with plasma from acute $P$. vivax-infected patients containing IFNY-reduced cell susceptibility to ONNV infection. Of note, the biological effects of IFNy are mediated conventionally through the activation of the JAK/STAT pathway (55). We postulate that the attenuation of ONNV infection by Plasmodium-induced IFN- $\gamma$ observed in mouse models could be translated to a real coinfection scenario in endemic populations given that the JAK/ STAT signaling pathway between humans and mice is highly conserved $(56,57)$. This suggests that similar downstream effector proteins could be involved in the IFNy-mediated restriction of alphavirus infection.

A recent study (58) showed that Plasmodium infection protected mice from Ebola virus (EBOV)-induced mortality via up-regulation of IFNy, supporting field reports where coinfected patients by EBOV and $P$. falciparum displayed increased survival rates (59). Conversely, two other murine coinfection models with respiratory viral pathogens such as murine pneumonia virus (PVM) and murine gammaherpesvirus 68 (MHV68) using nonlethal Plasmodium chabaudi and P. yoelii 17XNL have reported detrimental outcomes for the host such as increased viral loads in the lungs (60) and mortality due to severe anemia (61). These observations were linked to altered type I IFN production (60) and antiviral humoral responses (61) upon coinfection. Thus, the protective or detrimental effects of murine malaria on viral pathogens are likely associated to the modulation of distinct immune responses governing the control of different viral infections. Conversely, other protozoan parasites highly prevalent in the tropics and known to induce the upregulation of IFNy in response to infection such as Leishmania spp. (62), Toxoplasma spp. (63), or Trypanosoma spp. (64) could potentially display similar protective effects in coinfection settings. In line with this, mice infected with Trypanosoma brucei were shown to be resistant to $\mathrm{cu}$ taneous leishmaniasis through the induction of IFNy generating a hostile pro-inflammatory environment impairing L. major colonization of the skin (65).

Results in Fig 1B and E suggest the impairment of ONNV-induced joint pathologies upon Plasmodium infection. Interestingly, concurrent coinfection with PbA parasites did not affect viremia levels but significantly reduced the major peak of footpad swelling at 6 dpi. This can be the result of two different mechanisms. First, the development of viremia in concurrently coinfected mice can be attributed to the absence of Plasmodium-induced IFNY in the early stages of ONNV infection in the footpads. It has been shown that the earliest IFNy production during blood-stage murine malaria only occurs after $24 \mathrm{~h}$ postparasite injection (35). During this period, footpad cells in concurrently coinfected mice are still susceptible to ONNV infection which results in viremia levels similar to control mice (Fig 1F). On the other hand, the suppression of joint swelling upon coinfection could be linked to the dysregulation of virusspecific CD4 T-cell responses, main drivers of joint inflammation at $6 \mathrm{dpi}$ (26) by malaria. It has been reported that murine Plasmodium infections impair the development of CD4 T-cell responses against heterologous antigens $(66,67)$. Thus, PbA infections could alter the establishment of virus-specific CD4 T-cell immunity resulting in decreased footpad swelling.

between ONNV, PbA(-4dpi)+ONNV, and Py17x(-4dpi)+ONNV groups were calculated using two-tailed Kruskal-Wallis and post hoc Dunn's tests $\left({ }^{\star} P<0.05 * \star P<0.01\right.$, $\star * \star P<0.001)$. 
A

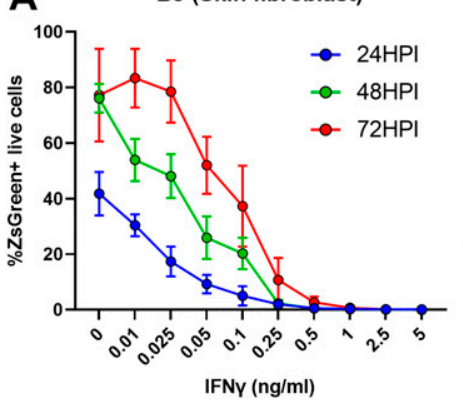

SW982 (Synoviocyte)

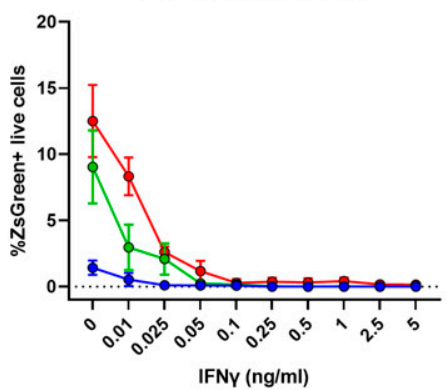

RD (Skeletal muscle)

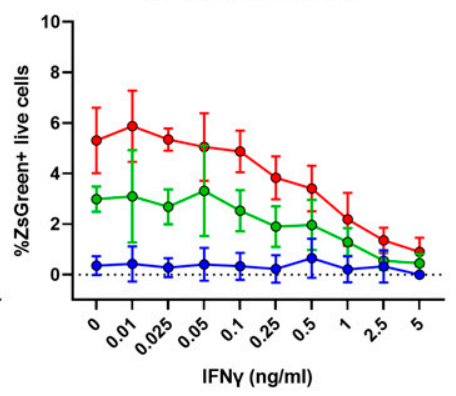

HPMEC (Endothelial)

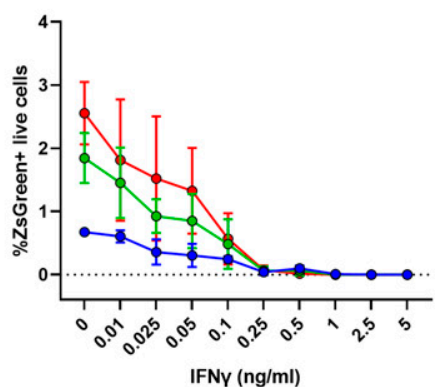

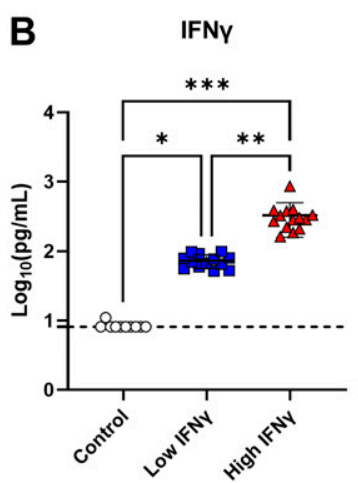

C

BJ (Skin fibroblast)

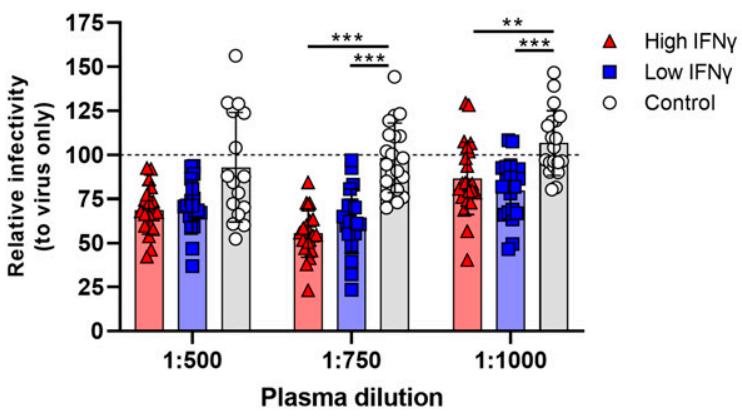

D

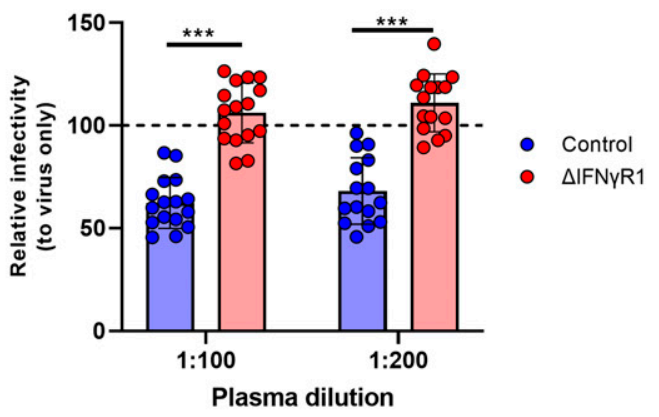

Figure 6. In vitro stimulation with human IFN $y$ or plasma from malaria patients reduces susceptibly to ONNV infection.

For (A), cells were treated with recombinant human IFNy for $24 \mathrm{~h}$ before ONNV infection at MOI 10. (A) ONNV infection rates in BJ, SW982, HPMEC, and RD at 24, 48, and 72 hpi. (B) IFNy levels in plasma of healthy controls $(H C, n=10)$, low $(n=13)$, and high $(n=14)$ IFNy responders. For (C), BJ cells were treated with pooled plasma dilutions from P. vivax-infected patients or healthy controls for $12 \mathrm{~h}$ before ONNV infection at MOI 1. For (D), control or $\Delta$ IFNyR1 HEK293T cells were treated with pooled plasma (1:100 or 1:200) from $P$. vivax-infected patients for $12 \mathrm{~h}$ before ONNV infection at MOI 1. Differences between three groups were calculated using two-tailed Kruskal-Wallis and post hoc Dunn's tests, differences between two groups were calculated using two-tailed Mann-Whitney $U$ test ( $\left.{ }^{*} P<0.05{ }^{* *} P<0.01,{ }^{* * *} P<0.001\right)$. Data are presented as mean \pm SD values and representative of two independent experiments.

The great success of malaria control programs during the last decade has been accompanied of by a sharp rise in the number of arbovirus infections worldwide (68). Our data highlight a possible causative link: that this phenomenon could be due in part to the loss of protective effects exerted by Plasmodium infections on alphavirus-induced pathologies that had hitherto masked the real burden of ONNV and other arbovirus infections. This may occur via two mechanisms: first, higher arbovirus case reporting because of more severe symptoms of arbovirus infections in the absence of malaria; second, and worse, increased arbovirus cases might reflect increased arbovirus transmissibility and/or fitness because of increased viral titers in the absence of malaria. Our data in this report will be of value in the fight against Plasmodium and ONNV infections in areas where both pathogens co-circulate, particularly highlighting the need for screening and clinical studies of underlying alphavirus infections in malaria intervention programs.

\section{Materials and Methods}

\section{Mice}

3- to 4-wk-old gender-matched wild-type (JAX \#000664), IFN ${ }^{-1-}$ (JAX \#002287), and IFNaR1 ${ }^{-1-}$ (JAX \#028288) mice in C57BL/6J background were used in this study. Animals were bred and maintained under specific pathogen-free conditions at the Biological Resource Centre of the Agency for Science, Technology, and Research, Singapore ( $A * S T A R)$.

\section{Viruses}

The IMTSSA/5163 ONNV isolate used in this study was obtained from an acute patient in Chad in 2004 (kindly provided by Marc Grandadam from the Unité de Virologie Tropicale, IMTSSA) (69). Fulllength infectious CDNA clones of the IMTSSA/5163 isolate were used to generate ONNV variants expressing the firefly luciferase gene (ONNV-Fluc) and ZsGreen protein (ONNV-ZsGreen) (70, 71). Viruses were propagated in Aedes albopictus C6/36 cell line (ATCC CRL1660) and purified by sucrose-gradient ultracentrifugation. Viral stock titers were determined by standard plaque assay using Vero E6 cells (ATCC CCL-81).

\section{Parasites}

P. yoelii 17XNL clone 1.1 (referred as Py17x) was used to induce selfresolving infections in mice (72). Lethal infections were induced by inoculation of $P$. berghei ANKA (PbA) (231cl1) expressing luciferase and GFP under the control of the ef1- $\alpha$ promoter (73). iRBCs were obtained by in vivo serial passage in C57BL/6J mice and were stored in Alsever's solution in liquid nitrogen. 


\section{Human cell lines}

BJ (ATCC CRL-2522), SW-982 (ATCC HTB-93), and RD (ATCC CCL-136) cells were grown in DMEM medium with $10 \%$ heat-inactivated FBS. HPMEC (\#3000; ScienCell) were grown in supplemented EC medium (\#1001; ScienCell). Cells were grown at $37^{\circ} \mathrm{C}$, relative humidity of $95 \%$, and $5 \% \mathrm{CO}_{2}$.

\section{Generation of $\triangle \mathrm{IFN} \gamma \mathrm{R} 1 \mathrm{HEK} 293 \mathrm{~T}$ cell line}

$\triangle$ IFNYR1 HEK293T cell line was generated by phosphorylating and annealing primers (5'-CACCGACATGAACCCTATCGTATAT-3') and (5'AAACATATACGATA GGGTTCATGTC-3') (NG_007394.1) using T4 Polynucleotide Kinase (NEB M0201S) in provided buffer supplemented with $1 \mathrm{mM}$ ATP (A2383; Sigma-Aldrich) at $37^{\circ} \mathrm{C}$ for $30 \mathrm{~min}$ followed by $5 \mathrm{~min}$ at $95^{\circ} \mathrm{C}$ and ramped down to $25^{\circ} \mathrm{C}$. Annealed primers were then ligated using Instant Sticky-end Ligase Master Mix (M0370; NEB) in PSB-CRIPSR (kindly gifted by $\mathrm{Dr}$ Gao and Dr Hu (74)) previously linearized using Esp3I (R0734S; NEB) in CutSmart Buffer (NEB) and purified using Nucleospin gel and PCR clean up kit (740609.50; Macherey-Nagel). HEK293T cells were co-transfected with pCMV(CAT)T7-SB100 (a gift from Zsuzsanna Izsvak, plasmid \# 34879; Addgene; RRID: Addgene_34879 (75)) and pSB-CRISPR (at 1:1 ratio) using Lipofectamine 2000 (11668019; Thermo Fisher Scientific) in Opti-MEM medium (31985070; Gibco) following the manufacturer's recommendations. $3 \mathrm{~d}$ post co-transfection, cells were passaged and cultured in complete media containing $1 \mu \mathrm{g} / \mathrm{ml}$ puromycin (P8833; Sigma-Aldrich). When control cells fully succumbed to puromycin selection, co-transfected cells were cultured in complete media and IFNYR1 expression was assessed by flow cytometry.

\section{ONNV infection and disease monitoring}

Mice were infected with ONNV by subcutaneous inoculation of $10^{6}$ PFU in $30 \mu \mathrm{l}$ of PBS in the ventral side of the right hind footpad. Viremia was monitored daily from 1 to $6 \mathrm{dpi}$ and thereafter on every alternate day until $12 \mathrm{dpi}$. Briefly, $10 \mu \mathrm{l}$ of blood collected from the tail of each mouse was mixed in $120 \mu$ of PBS supplemented with $10 \mu \mathrm{l}$ of citrate-phosphate-dextrose solution (Sigma-Aldrich). RNA isolation was performed using the QIAamp Viral RNA kit (QIAGEN) following the manufacturer's instructions with a final elution volume of $60 \mu \mathrm{l}$. $1 \mu \mathrm{l}$ of purified RNA was quantified by qRT-PCR using QuantiTect Probe RT-PCR (QIAGEN) as previously described (26). Joint swelling was measured for 2 wk post-ONNV inoculation as a function of height $\times$ width relative to measurements preinfection (relative increase) (26).

\section{Tissue viral load determination}

Ketamine xylazine-anesthetized mice $(150 \mathrm{mg} / \mathrm{kg}$ of ketamine, 10 $\mathrm{mg} / \mathrm{kg}$ of xylazine) were intracardially perfused with PBS and organs were collected in tubes containing zirconia beads (TOMY Digital Biology) and $1 \mathrm{ml} \mathrm{TRIzol}$ (Invitrogen) and stored at $-80^{\circ} \mathrm{C}$. To isolate RNA, tissues were thawed on ice and homogenized using the Bead Ruptor Elite (OMNI International) at a speed of $6 \mathrm{~m} / \mathrm{s}$ (3 cycles of lysis $\times 30 \mathrm{~s}$ ). Tissue lysates were then transferred to $1.5-\mathrm{ml}$
Eppendorf tubes and mixed with $230 \mu \mathrm{l}$ of chloroform and incubated at RT for 2 min. Samples were centrifuged at 12,000 g for $10 \mathrm{~min}$ at $4^{\circ} \mathrm{C}$ and recovered supernatants were transferred into clean Eppendorf Tubes and mixed with $70 \%$ ethanol (1:1 volume). RNA was purified using the RNeasy Mini Kit (QIAGEN) according to the manufacturer's protocol. Viral RNA copies were quantified by qRT-PCR as described above.

\section{In vivo virus tissue dissemination assay}

To quantify tissue viral load and virus dissemination in vivo, mice were inoculated with a firefly luciferase-tagged ONNV infectious clone (ONNV-Fluc) and virus dissemination was tracked using the IVIS Spectrum In Vivo Imaging System (Perkin-Elmer) (26). Animals were kept anesthetized during the experiment using an oxygen flow rate of 1 liter/minute with $2 \%$ isoflurane. Full-body shaved mice were subcutaneously injected with $100 \mu \mathrm{l}$ of D-luciferin potassium salt (Caliper Life sciences) diluted in PBS $(5 \mathrm{mg} / \mathrm{ml})$. Whole body and footpad bioluminescence readings were independently taken 7 min post-D-luciferin injection with a field of view (FOV) of $21.7 \mathrm{~cm}$ (ventral position) and $13.1 \mathrm{~cm}$ (dorsal position) for whole body (FOVD) and footpad (FOV-C) measurements, respectively. Two pictures were taken per FOV with exposure times set to "AUTO" and $60 \mathrm{~s}$. Regions of interest were drawn using the software Living Image 3.0 and total flux values (photons/second) were calculated. Readings of naive mice injected with D-luciferin were used for background subtraction.

\section{Plasmodium infection and disease monitoring}

Mice were infected with Py17x or PbA by i.p. injection of $10^{6}$ iRBC in Alsever's buffer. Parasitemia was monitored by flow cytometry as previously described (76) using a staining mix containing antimouse APC-tagged CD45 antibodies, $8 \mu \mathrm{M}$ dihydroethidium (SigmaAldrich), and $5 \mu \mathrm{g} / \mathrm{ml}$ Hoechst 33342 (Sigma-Aldrich). Successful infections were confirmed $4 \mathrm{~d}$ postinoculation.

\section{Isolation of footpad cells}

Homogenous cell suspensions were obtained for immune profiling of footpads of infected and naïve animals. Mice were culled by cervical dislocation and right paws were harvested and immediately placed in $4 \mathrm{ml}$ of digestion medium containing Collagenase IV (20 $\mathrm{g} / \mathrm{ml}$; Sigma-Aldrich), Dispase I (2 U/ml; Invitrogen), and DNase I (50 $\mathrm{\mu g} / \mathrm{ml}$; Roche Applied Science) mixed in RPMI medium complemented with 10\% FBS. Using forceps, footpads were deskinned and deboned to maximize digestion. Processed samples were placed on a shaker and incubated at $37^{\circ} \mathrm{C}$ at $100 \mathrm{rpm}$ (Biosan PSU10i) for $3 \mathrm{~h}$. After digestion, tissues were passed through a 40- $\mu \mathrm{m}$ cell strainer (Fisherbrand). Any remaining tissue trapped in the strainer was grinded using the top of a $1 \mathrm{ml}$ syringe plunger to maximize cell recovery. 1× Flow Cytometry Mouse Lysis Buffer (R\&D Systems) was used to lyse contaminating RBCs. Samples were resuspended in $1 \mathrm{ml}$ of complete RPMI, overlaid to $35 \% \mathrm{vol} / \mathrm{vol}$ Percoll (SigmaAldrich)/RPMI mixture, and centrifuged at 2,400 rpm for $20 \mathrm{~min}$ at $4^{\circ} \mathrm{C}$. Footpad cell pellets were washed and resuspended in appropriate volumes for counting using haemocytometers. 


\section{Profiling of immune and nonimmune cells by flow cytometry}

Cell suspensions were stained for viability using LIVE/DEAD Aqua dye (Life Technologies). Cells were washed and resuspended in $50 \mu$ of blocking buffer containing TruStain FCX PLUS (anti-mouse CD16/32, clone S17011E) antibody diluted in PBS and incubated in the dark for $15 \mathrm{~min}$ on ice. Conjugated anti-mouse antibodies CD45 (30-F11), CD9 (eBioKMC8), CD29 (HMß1-1), CD31 (390), and Integrin $\alpha 7$ (334908), Sca-1 (D7) were used to stain cell surface markers for 30 min on ice. Finally, cells were fixed with $50 \mu \mathrm{l}$ of eBioscience IC Fixation Buffer (Thermo Fisher Scientific) for 5 min and acquired using a 5-laser LSR II flow cytometer (BD Biosciences) with BD FACSDiva software. Data were analyzed with Flowjo v10.6.2 (Becton, Dickinson and Company).

\section{Dimensionality reduction analysis of flow cytometry data}

Live $C D 45^{-}$singlets events were pregated and then randomly downsampled to a fixed number $(n=5,000)$ for each sample using Flowjo v10.7 (Becton, Dickinson and Company). Down-sampled files were concatenated and analyzed using UMAP for Dimension Reduction plug-in v3.1 using default parameters (number of nearest neighbours $=15$, minimum distance $=0.1$.

\section{In vivo IFN $\gamma$ neutralization}

Anti-mouse IFNy (0.5 mg per mouse, clone XMG1.2, Bio X Cell) was i.p. injected at 0-, 2-, and 4 d postparasite inoculation. Control groups were given rat IgG1 Isotype control ( $0.5 \mathrm{mg}$, clone TNP6A7, Bio X Cell) at similar time points as treatment groups.

\section{Cytokine/chemokine quantification by multiplexed bead-based immunoassays}

Cytokine and chemokine concentrations ( $\mathrm{pg} / \mathrm{ml}$ ) were quantified in footpad and serum samples. For footpad samples, animals were anesthetized with ketamine-xylazine and intracardially perfused with PBS. The right paw was cut at the ankle and placed in a gentleMACS M tube (Miltenyi) filled with $1.5 \mathrm{ml}$ of RIPA buffer ( $50 \mathrm{mM}$ Tris- $\mathrm{HCl}, \mathrm{pH} 7.4,1 \% \mathrm{NP}-40,0.25 \%$ sodium deoxycholate, $150 \mathrm{mM}$ $\mathrm{NaCl}$, and $1 \mathrm{mM}$ EDTA) complemented with $1 \times$ complete Protease Inhibitor Cocktail (Roche). Samples were lysed in a Xiril Dispomix Tissue Homogenizer, centrifuged and the supernatants transferred into clean 2-ml microcentrifuge tubes for sonication in a Branson Ultrasonics Sonifier S-450 (70\% intensity × 15 s). For serum samples, blood was collected from the retro-orbicular sinus using a glass Pasteur pipette and allowed to clot for $30 \mathrm{~min}$ at room temperature. Clotted blood was centrifuged at 14,000 rpm for serum isolation. Footpad lysates and serum samples were analyzed using the Cytokine \& Chemokine 36-Plex Mouse ProcartaPlex Panel 1A (Thermo Fisher Scientific) according to the manufacturer's protocol. Human plasma samples were analyzed using the Cytokine/Chemokine/ Growth Factor 45-plex Human ProcartaPlex Panel 1 (Thermo Fisher Scientific). Data were acquired with Luminex FLEXMAP 3D instrument (Millipore) using xPONENT 4.0 software and analyzed with BioPlex Manager 6.1.1 (Bio-Rad Laboratories).

\section{In vitro IFN $y$ treatment and ONNV infection}

Recombinant human IFNy (PHC4033; Gibco) diluted in supplemented DMEM at various concentrations was used to treat skin fibroblasts (BJ), synoviocytes (SW-982), skeletal muscle cells (RD), and endothelial cells (HPMEC) for $24 \mathrm{~h}$ before virus infection. Cells were washed with PBS and then infected with ONNV-ZsGreen virus at MOI of 10. Cells were harvested at 24-, 48-, and 72-h postinfection (hpi) and percentage of infection was quantified by flow cytometry.

\section{Data and statistical analyses}

Statistical analyses were performed using GraphPad Prism 8.4.3 (GraphPad Software). Data are presented as mean \pm SD unless otherwise specified. Nonparametric Mann-Whitney U statistical test was used to compute differences between two groups. Differences between three groups were calculated using two-tailed KruskalWallis and post hoc Dunn's tests. Values obtained for viremia, parasitemia, in vivo imaging, and cytokine/chemokines were logtransformed for representation purposes. P-values < 0.05 were considered statistically significant.

\section{Study approval}

Animal experiments were approved by the Institutional Animal Care and Use Committee (IACUC \#211635) of A*STAR in accordance with the guidelines of the Agri-Food and Veterinary Authority (AVA) and the National Advisory Committee for Laboratory Animal Research of Singapore (NACLAR). Plasma samples from febrile $P$. vivax-infected patients from Mae Sot, Thailand, were collected and tested in accordance with protocols approved by the University of Oxford Tropical Research Ethics Committee (OXTREC 17-11) and the Ethics Committee of the Faculty of Tropical Medicine at Mahidol University (MUTM 2008-215). Written informed consent was received before participation.

\section{Supplementary Information}

Supplementary Information is available at https://doi.org/10.26508/lsa 202101272.

\section{Acknowledgements}

The authors would like to thank Dr Carla Claser for critical discussion and valuable suggestions on the study. We also thank the SIgN Flow Cytometry Core and SIgN Mouse Core for assistance with cytometry analyses and support in animal breeding, respectively. We thank Wilson How from the SIgN Immunomonitoring platform for his support in the multiplexed bead-based immunoassays. We also thank Professor Andres Merits from the University of Tartu for providing the tagged ONNV infectious clones used in this study. The study was supported by a core research grant provided to A*STAR Infectious Diseases Labs and Singapore Immunology Network by the Biomedical Research Council (BMRC) from the Agency for Science, Technology and Research ( $A^{*}$ STAR). A Torres-Ruesta is supported by the $A^{*}$ STAR Singapore International Graduate Award (SINGA) scholarship. Flow cytometry platform is supported by the Health and Biomedical Sciences (HBMS) Open Fund Shared Infrastructure Support Grant under the Immunomonitoring Service 
Platform project (NRF2017_SISFP09). The funders had no role in the study design, data collection and analysis, decision to publish, or preparation of the manuscript.

\section{Author Contributions}

A Torres-Ruesta: data curation, formal analysis, investigation, visualization, methodology, and writing-original draft, review, and editing.

T-H Teo: data curation, formal analysis, investigation, methodology, and writing-review and editing.

$\mathrm{Y}-\mathrm{H}$ Chan: formal analysis, investigation, methodology, and writing-review and editing.

SN Amrun: formal analysis, investigation, methodology, and writing-review and editing.

NK-W Yeo: formal analysis, investigation, methodology, and writing-review and editing.

CY-P Lee: formal analysis, investigation, methodology, and writing-review and editing.

SY-T Nguee: formal analysis, investigation, methodology, and writing-review and editing.

Mz Tay: formal analysis, investigation, methodology, and writing-review and editing.

F Nosten: formal analysis, investigation, methodology, and writing-review and editing.

S-W Fong: formal analysis, investigation, methodology, and writing-review and editing.

F-M Lum: formal analysis, investigation, methodology, and writing-review and editing.

G Carissimo: formal analysis, investigation, methodology, and writing-review and editing.

L Renia: conceptualization, supervision, and writing-original draft, review, and editing.

LFP Ng: conceptualization, resources, supervision, funding acquisition, project administration, and writing-original draft, review, and editing.

\section{Conflict of Interest Statement}

The authors declare that they have no conflict of interest.

\section{References}

1. Rezza G, Chen R, Weaver SC (2017) O'nyong-nyong fever: A neglected mosquito-borne viral disease. Pathog Glob Health 111: 271-275. doi:10.1080/20477724.2017.1355431

2. Powers AM, Brault AC, Shirako Y, Strauss EG, Kang W, Strauss JH, Weaver SC (2001) Evolutionary relationships and systematics of the alphaviruses. J Virol 75: 10118-10131. doi:10.1128/JVI.75.21.10118-10131.2001

3. Sanders EJ, Rwaguma EB, Kawamata J, Kiwanuka N, Lutwama JJ, Ssengooba FP, Lamunu M, Najjemba R, Were WA, Bagambisa G, et al (1999) O'nyong-nyong fever in south-central Uganda, 1996-1997: Description of the epidemic and results of a household-based seroprevalence survey. I Infect Dis 180: 1436-1443. doi:10.1086/315073

4. Williams MC, Woodall JP, Corbet PS, Gillett JD (1965) O'nyong-Nyong fever: An epidemic virus disease in East Africa. 8. Virus isolations from
Anopheles mosquitoes. Trans R Soc Trop Med Hyg 59: 300-306. doi:10.1016/0035-9203(65)90012-x

5. Haddow AJ, Davies CW, Walker AJ (1960) O'nyong-nyong fever: An epidemic virus disease in East Africa 1. Introduction. Trans $R$ Soc Trop Med Hyg 54: 517-522. doi:10.1016/0035-9203(60)90025-0

6. Rwaguma EB, Lutwama JJ, Sempala SD, Kiwanuka N, Kamugisha J, Okware S, Bagambisa G, Lanciotti R, Roehrig JT, Gubler DJ (1997) Emergence of epidemic O'nyong-nyong fever in southwestern Uganda, after an absence of 35 years. Emerg Infect Dis 3: 77. doi:10.3201/eid0301.970112

7. Posey DL, O'Rourke T, Roehrig JT, Lanciotti RS, Weinberg M, Maloney S (2005) O'Nyong-nyong fever in West Africa. Am J Trop Med Hyg 73: 32. doi:10.4269/ajtmh.2005.73.1.0730032

8. LaBeaud AD, Banda T, Brichard J, Muchiri EM, Mungai PL, Mutuku FM, Borland E, Gildengorin G, Pfeil S, Teng CY, et al (2015) High rates of O'nyong nyong and Chikungunya virus transmission in coastal Kenya. PLoS Negl Trop Dis 9: e0003436. doi:10.1371/journal.pntd.0003436

9. Clements TL, Rossi CA, Irish AK, Kibuuka H, Eller LA, Robb ML, Kataaha P, Michael NL, Hensley LE, Schoepp RJ (2019) Chikungunya and O'nyongnyong viruses in Uganda: Implications for diagnostics. Open Forum Infect Dis 6: ofz001. doi:10.1093/ofid/ofz001

10. Baba $\mathrm{M}$, Logue $\mathrm{CH}$, Oderinde B, Abdulmaleek $\mathrm{H}$, Williams J, Lewis J, Laws TR, Hewson R, Marcello A, D'Agaro P (2013) Evidence of arbovirus coinfection in suspected febrile malaria and typhoid patients in Nigeria. J Infect Dev Ctries 7: 51-59. doi:10.3855/jidc.2411

11. Kinimi E, Patrick BN, Misinzo G (2018) Serological evidence of chikungunya and malaria co-infection among febrile patients seeking health care in district, Tanzania. Tanzania J Health Res 20: 1-8. doi:10.4314/thrb.v20i4.1

12. Mostafavi H, Abeyratne E, Zaid A, Taylor A (2019) Arthritogenic alphavirus-induced immunopathology and targeting host inflammation as A therapeutic strategy for alphaviral disease. Viruses 11: 290. doi:10.3390/v11030290

13. Assunção-Miranda I, Cruz-Oliveira C, Da Poian AT (2013) Molecular mechanisms involved in the pathogenesis of -induced arthritis. Biomed Res Int 2013: 973516. doi:10.1155/2013/973516

14. Septembre-Malaterre A, Bedoui Y, Giry C, Gasque P, Guiraud P, Sélambarom J (2021) Quercetin can reduce viral RNA level of O'nyongnyong virus and resulting innate immune cytokine responses in cultured human synovial fibroblasts. Sci Rep 11: 6369. doi:10.1038/ s41598-021-85840-z

15. Clark IA (2007) The advent of the cytokine storm. Immunol Cell Biol 85 271-273. doi:10.1038/sj.icb.7100062

16. Clark IA, Alleva LM, Budd AC, Cowden WB (2008) Understanding the role of inflammatory cytokines in malaria and related diseases. Trav Med Infect Dis 6: 67-81. doi:10.1016/j.tmaid.2007.07.002

17. Kurup SP, Butler NS, Harty JT (2019) T cell-mediated immunity to malaria. Nat Rev Immunol 19: 457-471. doi:10.1038/s41577-019-0158-z

18. Rénia L, Howland SW, Claser C, Charlotte Gruner A, Suwanarusk R, Hui Teo T, Russell B, Ng LF (2012) Cerebral malaria: Mysteries at the bloodbrain barrier. Virulence 3: 193-201. doi:10.4161/viru.19013

19. Howland SW, Claser C, Poh CM, Gun SY, Rénia L (2015) Pathogenic CD8+ T cells in experimental cerebral malaria. Semin Immunopathol 37: 221-231. doi:10.1007/s00281-015-0476-6

20. Rénia L, Potter SM, Mauduit M, Rosa DS, Kayibanda M, Deschemin JC, Snounou G, Grüner AC (2006) Pathogenic T cells in cerebral malaria. Int J Parasitol 36: 547-554. doi:10.1016/j.ijpara.2006.02.007

21. Claser C, Nguee SYT, Balachander A, Howland SW, Becht E, Gunasegaran B, Hartimath SV, Lee AWQ, Ho JTT, Ong CB, et al (2019) Author Correction: Lung endothelial cell antigen cross-presentation to CD8+T cells drives malaria-associated lung injury. Nat Commun 10: 5066. doi:10.1038/ s41467-019-13025-4 
22. Wangoo A, Ganguly NK, Mahajan RC (1990) Immunosuppression in murine malaria: Suppressor role of macrophages and their products during acute and chronic Plasmodium berghei infection. APMIS 98: 407-414. doi:10.1111/j.1699-0463.1990.tb01051.x

23. Chemtai AK, Okelo GB (1989) Suppression of T-cell proliferative response in Plasmodium falciparum malaria patients-preliminary results. East Afr Med J 66: 787-791.

24. Theander TG, Bygbjerg IC, Andersen BJ, Jepsen S, Kharazmi A, Odum N (1986) Suppression of parasite-specific response in Plasmodium falciparum malaria. A longitudinal study of blood mononuclear cell proliferation and subset composition. Scand I Immunol 24: 73-81. doi:10.1111/j.1365-3083.1986.tb02071.x

25. Hviid L, Theander TG, Abu-Zeid YA, Abdulhadi NH, Jakobsen PH, Saeed BO, Jepsen S, Bayoumi RA, Jensen JB (1991) Loss of cellular immune reactivity during acute Plasmodium falciparum malaria. FEMS Microbiol Immunol 3: 219-227. doi:10.1111/j.1574-6968.1991.tb04218.x

26. Chan YH, Teo TH, Torres-Ruesta A, Hartimath SV, Chee RS, Khanapur S, Yong FF, Ramasamy B, Cheng P, Rajarethinam R, et al (2020) Longitudinal [18F]FB-IL-2 PET imaging to assess the immunopathogenicity of O'nyong-nyong virus infection. Front Immunol 11: 894. doi:10.3389/ fimmu.2020.00894

27. Lentscher AJ, McCarthy MK, May NA, Davenport BJ, Montgomery SA, Raghunathan K, McAllister N, Silva LA, Morrison TE, Dermody TS (2020) Chikungunya virus replication in skeletal muscle cells is required for disease development. J Clin Invest 130: 1466-1478. doi:10.1172/JCI129893

28. Gardner J, Anraku I, Le TT, Larcher T, Major L, Roques P, Schroder WA, Higgs S, Suhrbier A (2010) Chikungunya virus arthritis in adult wild-type mice. J Virol 84: 8021-8032. doi:10.1128/JVI.02603-09

29. Sourisseau M, Schilte C, Casartelli N, Trouillet C, Guivel-Benhassine F, Rudnicka D, Sol-Foulon N, Le Roux K, Prevost MC, Fsihi H, et al (2007) Characterization of reemerging chikungunya virus. PLoS Pathog 3: e89. doi:10.1371/journal.ppat.0030089

30. Halfon S, Abramov N, Grinblat B, Ginis I (2011) Markers distinguishing mesenchymal stem cells from fibroblasts are downregulated with passaging. Stem Cells Dev 20: 53-66. doi:10.1089/scd.2010.0040

31. Gabrielis K (2012) Surface markers distinguishing mesenchymal stem cells from fibroblasts. Acta Med Lituanica 19: 75-79. doi:10.6001/ actamedica.v19i2.2313

32. Kafadar KA, Yi L, Ahmad Y, So L, Rossi F, Pavlath GK (2009) Sca-1 expression is required for efficient remodeling of the extracellular matrix during skeletal muscle regeneration. Dev Biol 326: 47-59. doi:10.1016/j.ydbio.2008.10.036

33. Mierzejewski B, Archacka K, Grabowska I, Florkowska A, Ciemerych MA, Brzoska E (2020) Human and mouse skeletal muscle stem and progenitor cells in health and disease. Semin Cell Dev Biol 104: 93-104. doi:10.1016/j.semcdb.2020.01.004

34. Yao Z, Liu H, Yang M, Bai Y, Zhang B, Wang C, Yan Z, Niu G, Zou Y, Li Y (2020) Bone marrow mesenchymal stem cell-derived endothelial cells increase capillary density and accelerate angiogenesis in mouse hindlimb ischemia model. Stem Cell Res Ther 11: 221. doi:10.1186/s13287020-01710-x

35. De Souza JB, Williamson KH, Otani T, Playfair JH (1997) Early gamma interferon responses in lethal and nonlethal murine blood-stage malaria. Infect Immun 65: 1593-1598. doi:10.1128/IAI.65.5.1593-1598.1997

36. Mitchell AJ, Hansen AM, Hee L, Ball HJ, Potter SM, Walker JC, Hunt NH (2005) Early cytokine production is associated with protection from murine cerebral malaria. Infect Immun 73: 5645-5653. doi:10.1128/ IAI.73.9.5645-5653.2005

37. Miller JL, Sack BK, Baldwin M, Vaughan AM, Kappe SHI (2014) Interferonmediated innate immune responses against malaria parasite liver stages. Cell Rep 7: 436-447. doi:10.1016/j.celrep.2014.03.018

38. Kang S, Brown HM, Hwang S (2018) Direct antiviral mechanisms of interferon-gamma. Immune Netw 18: e33. doi:10.4110/in.2018.18.e33
39. He X, Xia L, Tumas KC, Wu J, Su XZ (2020) Type I interferons and malaria: A double-edge sword against a complex parasitic disease. Front Cell Infect Microbiol 10: 594621. doi:10.3389/fcimb.2020.594621

40. Sebina I, Haque A (2018) Effects of type I interferons in malaria. Immunology 155: 176-185. doi:10.1111/imm.12971

41. Mooney JP, Wassmer SC, Hafalla JC (2017) Type I interferon in malaria: A balancing act. Trends Parasitol 33: 257-260. doi:10.1016/j.pt.2016.12.010

42. Couderc T, Chrétien F, Schilte C, Disson O, Brigitte M, Guivel-Benhassine F, Touret Y, Barau G, Cayet N, Schuffenecker I, et al (2008) A mouse model for chikungunya: Young age and inefficient type-I interferon signaling are risk factors for severe disease. PLoS Pathog 4: e29. doi:10.1371/ journal.ppat.0040029

43. Seymour RL, Rossi SL, Bergren NA, Plante KS, Weaver SC (2013) The role of innate versus adaptive immune responses in a mouse model of O'nyong-nyong virus infection. Am J Trop Med Hyg 88: 1170-1179. doi:10.4269/ajtmh.12-0674

44. Crisler WJ, Lenz LL (2018) Crosstalk between type I and II interferons in regulation of myeloid cell responses during bacterial infection. Curr Opin Immunol 54: 35-41. doi:10.1016/j.coi.2018.05.014

45. Rayamajhi M, Humann J, Kearney S, Hill KK, Lenz LL (2010) Antagonistic crosstalk between type I and II interferons and increased host susceptibility to bacterial infections. Virulence 1: 418-422. doi:10.4161/ viru.1.5.12787

46. Gough DJ, Messina NL, Hii L, Gould JA, Sabapathy K, Robertson AP, Trapani JA, Levy DE, Hertzog PJ, Clarke CJ, et al (2010) Functional crosstalk between type I and II interferon through the regulated expression of STAT1. PLOS Biol 8: e1000361. doi:10.1371/journal.pbio.1000361

47. Schroder K, Hertzog PJ, Ravasi T, Hume DA (2004) Interferon-gamma: An overview of signals, mechanisms and functions. J Leukoc Biol 75: 163-189. doi:10.1189/jlb.0603252

48. Young AR, Locke MC, Cook LE, Hiller BE, Zhang R, Hedberg ML, Monte KJ, Veis DJ, Diamond MS, Lenschow DJ (2019) Dermal and muscle fibroblasts and skeletal myofibers survive chikungunya virus infection and harbor persistent RNA. PLoS Pathog 15: e1007993. doi:10.1371/ journal.ppat.1007993

49. Burdeinick-Kerr R, Griffin DE (2005) Gamma interferon-dependent, noncytolytic clearance of virus infection from neurons in vitro. J Virol 79: 5374-5385. doi:10.1128/JVI.79.9.5374-5385.2005

50. Burdeinick-Kerr R, Govindarajan D, Griffin DE (2009) Noncytolytic clearance of virus infection from neurons by gamma interferon is dependent on Jak/STAT signaling. J Virol 83: 3429-3435. doi:10.1128/ JVI.02381-08

51. Schilte C, Couderc T, Chretien F, Sourisseau M, Gangneux N, GuivelBenhassine F, Kraxner A, Tschopp J, Higgs S, Michault A, et al (2010) Type I IFN controls chikungunya virus via its action on nonhematopoietic cells. J Exp Med 207: 429-442. doi:10.1084/jem.20090851

52. Ryman KD, Klimstra WB, Nguyen KB, Biron CA, Johnston RE (2000) Alpha/ beta interferon protects adult mice from fatal Sindbis virus infection and is an important determinant of cell and tissue tropism. I Virol 74: 3366-3378. doi:10.1128/jvi.74.7.3366-3378.2000

53. Metzemaekers M, Vanheule V, Janssens R, Struyf S, Proost P (2017) Overview of the mechanisms that may contribute to the non-redundant activities of interferon-inducible CXC chemokine receptor 3 ligands. Front Immunol 8: 1970. doi:10.3389/fimmu.2017.01970

54. Liu Y, Cai Y, Liu L, Wu Y, Xiong X (2018) Crucial biological functions of CCL7 in cancer. PeerJ 6: e4928. doi:10.7717/peerj.4928

55. Gough DJ, Levy DE, Johnstone RW, Clarke CJ (2008) IFNgamma signalingdoes it mean JAK-STAT? Cytokine Growth Factor Rev 19: 383-394. doi:10.1016/j.cytogfr.2008.08.004

56. Durbin JE, Hackenmiller R, Simon MC, Levy DE (1996) Targeted disruption of the mouse Stat1 gene results in compromised innate immunity to viral disease. Cell 84: 443-450. doi:10.1016/s0092-8674(00)81289-1 
57. Meraz MA, White JM, Sheehan KC, Bach EA, Rodig SJ, Dighe AS, Kaplan DH, Riley JK, Greenlund AC, Campbell D, et al (1996) Targeted disruption of the Stat1 gene in mice reveals unexpected physiologic specificity in the JAK-STAT signaling pathway. Cell 84: 431-442. doi:10.1016/s0092-8674(00) 81288-X

58. Rogers KJ, Shtanko O, Vijay R, Mallinger LN, Joyner CJ, Galinski MR, Butler NS, Maury W (2020) Acute Plasmodium infection promotes interferongamma-dependent resistance to Ebola virus infection. Cell Rep 30: 4041-4051.e4. doi:10.1016/j.celrep.2020.02.104

59. Rosenke K, Adjemian J, Munster VJ, Marzi A, Falzarano D, Onyango CO, Ochieng M, Juma B, Fischer RJ, Prescott JB, et al (2016) Plasmodium parasitemia associated with increased survival in Ebola virus-infected patients. Clin Infect Dis 63: 1026-1033. doi:10.1093/cid/ciw452

60. Edwards CL, Zhang V, Werder RB, Best SE, Sebina I, James KR, Faleiro RJ, de Labastida Rivera F, Amante FH, Engwerda CR, et al (2015) Coinfection with blood-stage Plasmodium promotes systemic type I interferon production during pneumovirus infection but impairs inflammation and viral control in the lung. Clin Vaccin Immunol 22: 477-483. doi:10.1128/ CVI.00051-15

61. Matar CG, Anthony NR, O'Flaherty BM, Jacobs NT, Priyamvada L, Engwerda CR, Speck SH, Lamb TJ (2015) Co-infection with malaria suppresses anti-parasitic humoral immunity. PLoS Pathog 11: e1004858. doi:10.1371/journal.ppat.1004858

62. Kima PE, Soong L (2013) Interferon gamma in leishmaniasis. Front Immunol 4: 156. doi:10.3389/fimmu.2013.00156

63. Sturge CR, Yarovinsky F (2014) Complex immune cell interplay in the gamma interferon response during Toxoplasma gondii infection. Infect Immun 82: 3090-3097. doi:10.1128/IAl.01722-14

64. Rodrigues AA, Saosa JS, da Silva GK, Martins FA, da Silva AA, Souza Neto CP, Horta CV, Zamboni DS, da Silva JS, Ferro EA, et al (2012) IFN- $\gamma$ plays a unique role in protection against low virulent Trypanosoma cruzi strain. PLoS Negl Trop Dis 6: e1598. doi:10.1371/journal.pntd.0001598

65. Pereira L, Oliveira F, Townsend S, Metangmo S, Meneses C, Moore IN, Brodskyn Cl, Valenzuela JG, Magez S, Kamhawi S (2018) Coinfection with trypanosoma brucei confers protection against cutaneous leishmaniasis. Front Immunol 9: 2855. doi:10.3389/fimmu.2018.02855

66. Millington OR, Di Lorenzo C, Phillips RS, Garside P, Brewer JM (2006) Suppression of adaptive immunity to heterologous antigens during Plasmodium infection through hemozoin-induced failure of dendritic cell function. J Biol 5: 5. doi:10.1186/jbiol34
67. Millington OR, Gibson VB, Rush CM, Zinselmeyer BH, Phillips RS, Garside P, Brewer JM (2007) Malaria impairs T cell clustering and immune priming despite normal signal 1 from dendritic cells. PLoS Pathog 3: 1380-1387. doi:10.1371/journal.ppat.0030143

68. Ferguson NM (2018) Challenges and opportunities in controlling mosquito-borne infections. Nature 559: 490-497. doi:10.1038/s41586018-0318-5

69. Bessaud M, Peyrefitte CN, Pastorino BA, Gravier P, Tock F, Boete F, Tolou HJ, Grandadam M (2006) O'nyong-nyong Virus, Chad. Emerg Infect Dis 12 1248-1250. doi:10.3201/eid1208.060199

70. Carissimo G, Ng LFP (2019) Understanding molecular pathogenesis with chikungunya virus research tools. Curr Top Microbiol Immunol 1-21. doi:10.1007/82_2019_158

71. Pohjala L, Utt A, Varjak M, Lulla A, Merits A, Ahola T, Tammela P (2011) Inhibitors of entry and replication identified with a stable Chikungunya replicon cell line and virus-based assays. PLoS One 6: e28923. doi:10.1371/journal.pone.0028923

72. Weiss WR, Good MF, Hollingdale MR, Miller LH, Berzofsky JA (1989) Genetic control of immunity to Plasmodium sporozoites. J Immunol 143 : 4263-4266.

73. van Dijk MR, Waters AP, Janse CJ (1995) Stable transfection of malaria parasite blood stages. Science 268: 1358-1362. doi:10.1126/science. 7761856

74. Hu K, Li Y, Wu W, Chen H, Chen Z, Zhang Y, Guo Y, Dong Y (2018) Highperformance gene expression and knockout tools using sleeping beauty transposon system. Mob DNA 9: 33. doi:10.1186/s13100-018-0139-y

75. Mátés L, Chuah MK, Belay E, Jerchow B, Manoj N, Acosta-Sanchez A, Grzela DP, Schmitt A, Becker K, Matrai J, et al (2009) Molecular evolution of a novel hyperactive Sleeping Beauty enables robust stable gene transfer in vertebrates. Nat Genet 41: 753-761. doi:10.1038/ng.343

76. Malleret B, Claser C, Ong AS, Suwanarusk R, Sriprawat K, Howland SW, Russell B, Nosten F, Rénia L (2011) A rapid and robust tri-color flow cytometry assay for monitoring malaria parasite development. Sci Rep 1: 118. doi:10.1038/srep00118

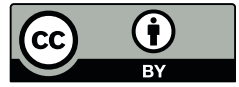

License: This article is available under a Creative Commons License (Attribution 4.0 International, as described at https://creativecommons.org/ licenses/by/4.0/). 